\title{
Anabases
}

ANABASES Traditions et réceptions de l'Antiquité

29 | 2019

Varia

\section{Jessica PRIESTLEY, Vasiliki ZALI}

( éd.),

Brill's Companion to the Reception of Herodotus in Antiquity and Beyond

\section{Olivier Gengler}

\section{(2) OpenEdition}

Journals

Édition électronique

URL : https://journals.openedition.org/anabases/9501

DOI : 10.4000/anabases.9501

ISSN : 2256-9421

Éditeur

E.R.A.S.M.E.

\section{Édition imprimée}

Date de publication : 14 avril 2019

Pagination : 392-395

ISSN : 1774-4296

\section{Référence électronique}

Olivier Gengler, « Jessica PRIEStLey, Vasiliki zali (éd.), Brill's Companion to the Reception of Herodotus in Antiquity and Beyond », Anabases [En ligne], 29 | 2019, mis en ligne le 14 avril 2019, consulté le 06 novembre 2021. URL : http://journals.openedition.org/anabases/9501 ; DOI : https://doi.org/10.4000/ anabases.9501 

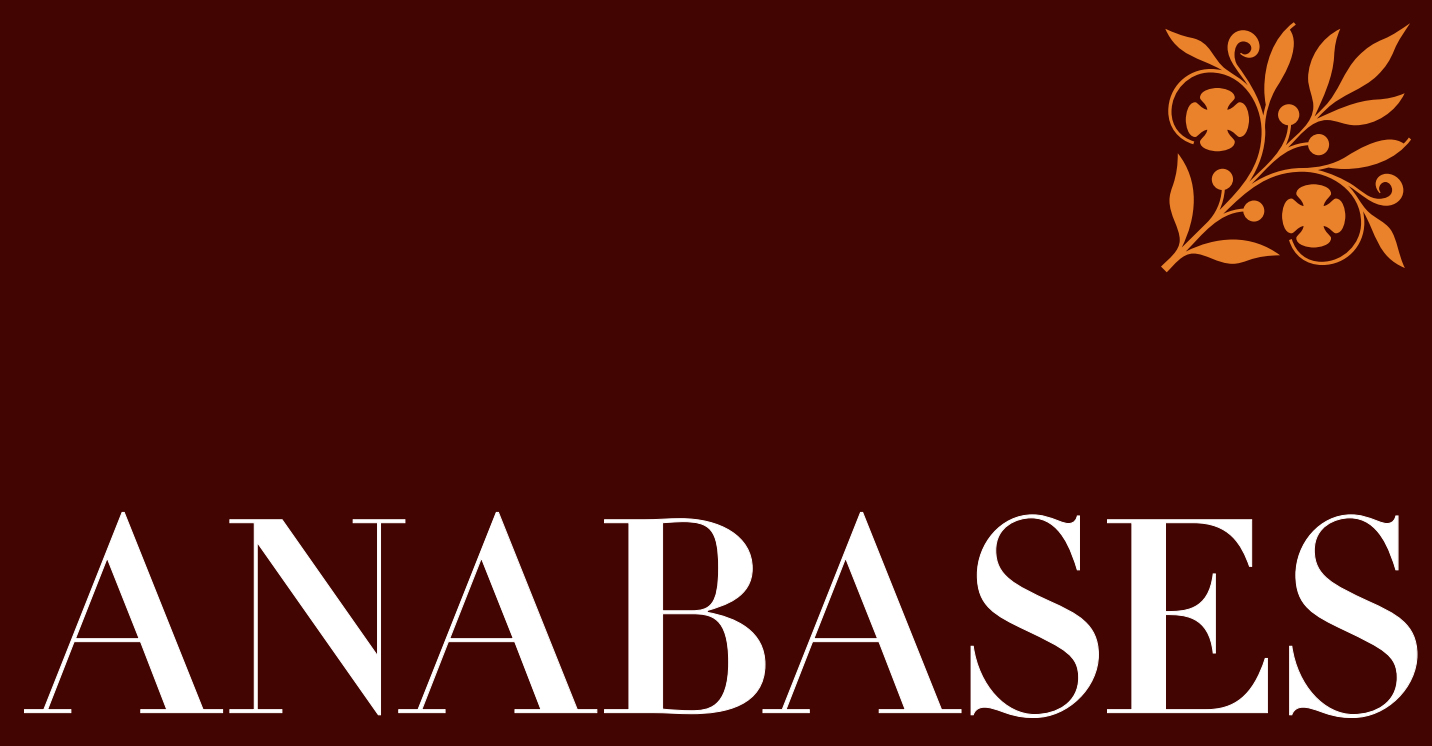

Traditions et Réceptions de l'Antiquité

\section{No29 \\ 2019}

Juliette Ernst Sculptures onctueuses de Meekyoung Shin Théâtre antique et travaux savants dans la Première modernité L'Antiquité dans la peinture (17911880) Réception d'Ovide Pierre Grimal 
ANABASES

Traditions et Réceptions de l'Antiquité

Revue de l'équipe de recherche E.R.A.S.M.E.

Université Toulouse-Jean Jaurès (UT2J)

Anabases dispose d'un Comité de lecture international. Chaque article envoyé à la rédaction est soumis, une fois anonymisé, à l'expertise de deux spécialistes qui rendent un rapport écrit. Les deux rapports anonymisés sont transmis à l'auteur qui tient compte des observations en vue de la publication.

\section{Comité SCIEnTIFIQUe}

Germaine Aujac (université Toulouse-Jean Jaurès : histoire de la géographie et des sciences antiques)

Florence Bouchet (université Toulouse-Jean Jaurès : littérature médiévale)

Hinnerk BruHns (CNRS : histoire économique et sociale ancienne et contemporaine)

Paulo Butti de Lima (université de Bari : historiographie et réception de l'Antiquité)

Luciano CANFora (université de Bari : littérature et histoire anciennes, historiographie)

Giovanna Ceserani (Stanford University : histoire intellectuelle et historiographie de la tradition classique)

Temístocles Cezar (université de Porto Alegre : historiographie moderne)

Serafina Сиомо (University of London, Birkbeck College : histoire des mathématiques et des sciences)

Paul Demont (université de Paris Sorbonne : philologie grecque et héritage classique)

Marie-Laurence Desclos (université de Grenoble II : philosophie de l'Antiquité)

Olivier Devillers (université de Bordeaux 3 - Michel-de-Montaigne : littérature et historiographie latines)

Andrea Giardina (Istituto italiano di scienze umane : histoire du monde romain et de ses réceptions)

Ève Gran-Aymerich (aibl : histoire de l'archéologie et des transferts culturels)

François HaRTog (EHEss : historiographie ancienne et moderne)

Geneviève Hoffmann (université de Picardie : histoire des mondes grecs)

Christian JACOB (CNRS/EHEss : histoire comparée et épistémologie des savoirs)

Suzanne Marchand (Louisiana State University : histoire du classicisme et de l'orientalisme)

Wilfried Nippel (Humboldt Universität Berlin : histoire et historiographie de l'Antiquité)

Sylvie Pittia (université de Paris I-Panthéon Sorbonne : histoire et historiographie du monde romain)

Stéphane Ratri (université de Franche-Comté - Besançon : philologie et héritage latin)

Comité de RÉdaction

Jacques Alexandropoulos, Marielle de Béchillon, Corinne Bonnet, Laurent Bricault, Clément Bur,

Philippe Foro, Adeline Grand-Clément, Anne-Hélène Klinger-Dollé, Véronique Krings,

Thibaud Lanfranchi, Claudine Leduc, Pascal Payen, Grégory Reimond, Catherine Valenti

Éditeur RESPonsable

Pascal PAYen

Université Toulouse-Jean Jaurès (UT2J)

SECRÉTARIAT DE RÉDACTION

Anthony Andurand / Clément Bertau-Courbières / Corinne Bonnet / Clément Bur /

Adeline Grand-Clément / Anne-Hélène Kuinger-Dollé / Véronique Krings /

Catherine Valenti (université Toulouse-Jean Jaurès) / Noémie VillacÈQue (université de Reims)

Sites Web

http://plh.univ-tlse2.fr

Revues.org : http://anabases.revues.org

Aвonnement et vente aU numéro

Éditions De Boccard - 4, rue de Lanneau - 75005 Paris

info@deboccard.com - www.deboccard.com

Tél. : 0033/(0)143260037 - Fax : 0033/(0)143548583 




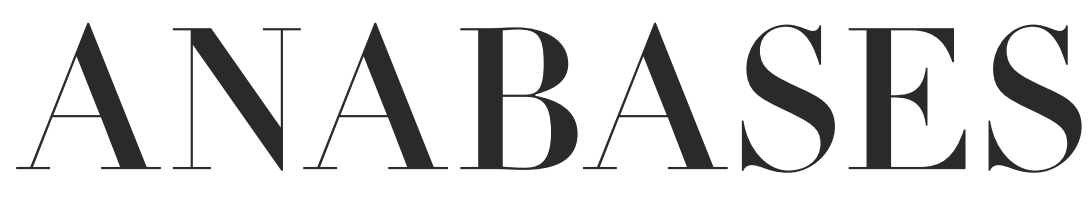

Traditions et Réceptions de l'Antiquité

$$
\begin{aligned}
& N \circ 29 \\
& 2019
\end{aligned}
$$

\section{E.R.A.S.M.E.}

Université Toulouse - Jean Jaurès 



\section{Sommaire}

$\mathrm{N}^{\circ} 29-2019$

\section{Historiographie et identités culturelles}

Ilse Hilbold

Les archives d'une bibliographe des sciences de l'Antiquité :

Juliette Ernst et la fabrique des relations internationales . . . . . . . . . I I3

Vivien LONGHI

La crise, une notion politique héritée des Grecs ? . . . . . . . . . . . 2I

Mireille Lacave-Allemand et Michel Lacave,

L’Antiquité dans la peinture en France, I79I-I880 :

une analyse quantitative à travers les Salons et les Prix de Rome . . . . . 37

Tiphaine Besnard

Du Weathering Project aux autoportraits en Venus :

Les sculptures onctueuses et savonneuses de Meekyoung Shin . . . . . 7 I

\section{Traditions du patrimoine antique}

Dossier dirigé par Pascale Paré-Rey et Malika Bastin-Hammou,

“La réception du théâtre antique dans les travaux savants de l’Europe

de la Première modernité »

Malika Bastin-Hammou et Pascale Paré-Rey

“ La réception du théâtre antique dans les travaux savants

de l'Europe de la Première modernité » . . . . . . . . . . . . . . . . 89

Kevin Bovier

Rétablir la métrique de Térence au $\mathrm{XvI}^{\mathrm{e}}$ siècle :

le cas du Iudicium de Glaréan (1540) . . . . . . . . . . . . . . . . . . . . . . 93 
Brice Denoyer

L'héritage de la métrique antique

dans l'alexandrin français au xvie siècle . . . . . . . . . . . . . IO7

Giovanna Di Martino

Vittorio Alfieri's tormented relationship with Aeschylus:

Agamennone between Tradition and Innovation . . . . . . . . . . . . . . . I2I

Marco Duranti

La condanna del prologo diegetico euripideo dagli scoli antichi

ai trattati del Cinquecento . . . . . . . . . . . . . . .

Rosario López Gregoris

L'influence de l' Arte nuevo de hacer comedias de Lope de Vega

dans l'usage des modèles classiques latins en Espagne

pendant le Siècle d'or et le Baroque » . . . . . . . . . . . . . . . . I49

Cressida Ryan

Sophoclean scholarship as a tool

to interpret eighteenth-century England . . . . . . . . . . . . . . г 6 I

Záviš ŠumaN

Axiologie critique de La Mesnardière . . . . . . . . . . . . . . . . . . . . I79

\section{Archéologie des savoirs}

Dossier dirigé par Cristina Noacco

“2000 ans déjà... Aspects de la réception d'Ovide » . . . . . . . . . . . I93

\section{La réception d'Ovide au Moyen Âge}

Jean-Marie Fritz et Cristina NoAcco

Lire Ovide au xiI ${ }^{\mathrm{e}}$ siècle : Arnoul d'Orléans

commentateur des Métamorphoses . . . . . . . . . . . . . . . $\quad{ }_{195}$

Franck Coulson

Le mythe de Pythagore dans le commentaire

“Vulgate » des Métamorphoses . . . . . . . . . . . . . . . . . . . . . . 2I

Marylène Possamaï

Comment éditer l'Ovide moralisé :

le problème de la mise en page du manuscrit Rouen Bm O.4 . . . . . . . 225

Anneliese Pollock Renck

Les Hérö̈des à la fin du Moyen Âge : pour une définition élargie de l'acte traducteur . . . . . . . . . . . . . . . . . . 239 
II. La réception d'Ovide à l'époque moderne

Fátima Díez Platas et Patricia Meilán Jácome

Le poète dans son œuvre. Ovide dans les images des Fasti

et des Tristia entre les Xv et $\mathrm{xvI}^{\mathrm{e}}$ siècles . . . . . . . . . . . . . . . . . 255

Ana Paula Rebelo Correia

Les représentations des Métamorphoses d'Ovide

dans les azulejos portugais. Influence des modèles gravés français . . . . 269

Sarah ReY

Figures d'Orphée au cinéma . . . . . . . . . . . . . . . . . . . 277

\section{Actualités et débats}

Marine LE BAIL

La modernité littéraire serait-elle affaire d'Antiquité(s) ?

Euvres \& Critiques: La contribution de l'archéologie à la genèse

de la littérature moderne, XLII, I, René Sternke dir., 20I7, 338 p. . . . . . . 2 29I

\section{Lire, relire la bibliothèque des sciences de l'Antiquité}

Éric Morvillez

“Les Horti Tauriani de Pierre Grimal

ou les prémices des Jardins romains » . . . . . . . . . . . . . . . . 30I

Pierre Grimal

“Les Horti Tauriani. Étude topographique sur la région

de la Porte Majeur ",MEFRA, tome 53, rg36. p. 25o-286 . . . . . . . . . . . 3 3i3

\section{L'atelier de l'histoire : chantiers historiographiques}

L'Antiquité au musée (coordonné par Adeline Grand-Clément) (6)

Aurélie Rodes, Catherine Valenti

Les Gaulois au musée . . . . . . . . . . . . . . . . . . . .

355

L'Atelier des doctorants (coordonné par Adeline Grand-Clément) (16)

Andrea Avalli

La question étrusque dans l'Italie fasciste $\ldots \ldots \ldots$. . . . . . . 360 
Droit et réception de l'Antiquité

(coordonné par Marielle de Béchillon et Hélène Ménard) (6)

Entre Clio et Thémis. Entretien avec Dario Mantovani, réalisé par

Hélène Ménard (Maître de Conférences d'Histoire romaine, à l'Université

Paul Valéry - Montpellier III), le 22 juin 20I8, à l'occasion de la parution

aux Belles Lettres du livre Les juristes écrivains de la Rome antique.

Les ouvres des juristes comme littérature (juin 20ı8) et de la création

de la chaire “ Droit, culture et société de la Rome antique »

au Collège de France $\left(\mathrm{I}^{\mathrm{er}}\right.$ novembre $\left.20 \mathrm{I} 8\right) \ldots$. . . . . . . . . . . . .

\section{Comptes rendus}

Philippe Borgeaud et Sara Petrella

Le singe de l'autre.

Du sauvage américain à l'histoire comparée des religions (A. Guedon) . . $\quad 37 \mathrm{I}$

Roberta Casagrande-Kim, Samuel Thrope et Raquel Ukeles (éd.)

Romance and reason. Islamic transformations of the classical past

(Cl. Bertau-Courbières) . . . . . . . . . . . . . . . . . . . . .

Hinnerk BruHns

Max Webers historische Sozialökonomie.

L'économie de Max Weber entre histoire et sociologie (Th. Lanfranchi) . . 374

Andrea Cozzo

Riso e sorriso, e altre saggi sulla nonviolenza nella Grecia antica,

(Fr. Pr. Barone) . . . . . . . . . . . . . . . . . . . 377

Franz Cumont

Manichéisme (St. Ratti) . . . . . . . . . . . . . . . 378

Emmanuelle HÉnin et Valérie NAAs (dir.)

Le mythe de l'art antique (Cl. Evrard) . . . . . . . . . . . . . . . . . 380

Jacques Jouanna, Henri Lavagne, Alain Pasquier,

Véronique SchiLtz et Michel Zink (éd.)

Au-delà du Savoir : Les Reinach et le Monde des Arts (G. Hoffmann) . . . .

382

Mario Liverani

Imagining Babylon: The Modern Story of an Ancient City (C.Bonnet) . . . 386

Françoise-Hélène Massa-Pairault, Claude Pouzadoux (Dir.)

Géants et Gigantomachie entre Orient et Occident (C.Giovénal) . . . . . . .

Scott McGill, Joseph Pucci (éd.)

Classics renewed. Reception and Innovation in the Latin Poetry

ofLate Antiquity (S. Clément-Tarantino) . . . . . . . . . . . . . . . . . . . 
Maxwell T. PAule

Canidia, Rome's First Witch (C. Landrea) . . . . . . . . . . . . . . . . . . . 39г

Jessica Priestley, Vasiliki Zali (éd.)

Brill's Companion to the Reception of Herodotus in Antiquity

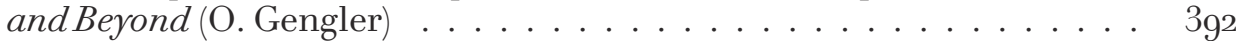

Salvatore QuAsimodo

La Lyre grecque $(\mathrm{M}$. Bianco) . . . . . . . . . . . . . . . . . . 395

Brett M. Rogers, Benjamin Eldon Stevens (éd.)

Classical Traditions in Modern Fantasy (M. Scapin) . . . . . . . . . . . . . 397

Maria Teresa Schettino et Céline UrLacher-Becht (dir.)

Ipse dixit. L'autorité intellectuelle des Anciens : affirmation,

appropriations, détournements (C. Psilakis) . . . . . . . . . . . . . 398

Guy G. Stroumsa

Religions d'Abraham : histoires croisées (D. Lorin) . . . . . . . . . . . . 400

Jean Yvonneau (éd.)

La Muse au long couteau. Critias, de la création littéraire

au terrorisme d'État (G. Hoffmann) . . . . . . . . . . . . . . 405

Résumés . . . . . . . . . . . . . . . . . . . . . 409

Index .............................. 423 



\section{Comptes rendus de lecture}



Philippe Borgeaud et Sara Petrella, Le singe de l'autre. Du sauvage américain à l'histoire comparée des religions, Genève, Éditions des Cendres, “Le monde dans une noix » II, 20I6, I23 p. +83 ill., 24 euros /ISBN 978-2-86742-258-4.

Lors d'un entretien, réalisé dans le cadre du séminaire de l'équipe ERASME et rapporté dans le numéro 2I d'Anabases, Christian Jacob explicitait les enjeux des comparatismes (Anabases 2I, 20I5, p. I97-2II). Àcette occasion, étaient rappelées la complexité et l'exigence des comparaisons historiques qui ne peuvent être menées qu'à l'issue d'une solide réflexion méthodologique. Le bel ouvrage que proposent Ph. Borgeaud et S. Petrella s'inscrit parfaitement dans cette démarche réflexive sur les pratiques comparatistes en général, sur l'histoire comparée des religions en particulier. Il se place également dans la lignée des travaux de Ph. Borgeaud, consacrés à l'histoire des religions en tant qu'objet d'étude. Les auteurs reviennent ainsi sur la longue “ genèse » (p. q) de la méthode comparative, appliquée à l'histoire des religions, en invitant le lecteur à visiter les « ateliers comparatistes » (p. 9) du père jésuite J. F. Lafitau et de l'éditeur J.-F. Bernard, associé au graveur B. Picart.

La démarche, le cadre théorique et le positionnement de $\mathrm{Ph}$. Borgeaud et de S. Petrella sont présentés avec beaucoup de clarté dans une riche introduction. Les auteurs rappellent, à la suite de G. Stroumsa, que le concept de religion, tel qu'on l'entend aujourd'hui, est une construction moderne. Ils insistent sur l'idée que l'histoire des religions est pensée, dès ses débuts, comme une histoire comparatiste puisque “les modernes, au xviI ${ }^{\mathrm{e}}$ siècle, découvrent l'histoire des religions en repensant le rapport entre la Bible et son environnement antique» (p. 9). Les auteurs reviennent ainsi sur les débuts de l'exégèse comparatiste et montrent comment l'Antiquité classique est mobilisée, aux côtés des Pères de l'Église, afin d'expliquer l'idolâtrie. Les comparaisons sont donc bien au cœur du propos et l'on pourrait les appréhender selon trois niveaux. Tout d'abord, l'enquête porte plus précisément sur deux ouvrages, publiés au début du xvıII ${ }^{\mathrm{e}}$ siècle : les Moeurs des sauvages amériquains comparées aux mours des premiers temps (Paris, I724) de Lafitau et les Cérémonies et coutumes religieuses de tous les peuples du monde (Amsterdam, I723 pour les deux premiers volumes), édité par Bernard. Ces deux publications sont rapprochées l'une de l'autre car elles abordent toutes deux les pratiques des "sauvages» en insistant notamment sur leurs gestes et sur les objets qu'ils fabriquent. Cependant, plus que les motifs de rapprochement, les auteurs tâchent d'analyser les différentes postures adoptées, “dans leur irrésoluble contradiction » (p. I5). En outre, ils s'intéressent à la méthode comparative élaborée dans chacun de ces écrits. Ils mettent en évidence les différents regards portés sur une altérité plurielle mettant en rapport les "sauvages ", les Modernes mais aussi les Anciens. Enfin, la confrontation des textes 
et des nombreuses images qui composent les deux livres pourrait constituer un troisième niveau de comparaison.

Dès les premières lignes, les auteurs rappellent l'importance fondamentale dans le contexte intellectuel, politique et religieux actuel, d'une attitude réflexive sur les sciences humaines en général, sur l'histoire des religions en particulier. Leur démarche se place dans la lignée du perspectivisme, défini par l'ethnologue E. Viveiros de Castro. Ils assument ainsi une " posture de renoncement àtoute prétention universelle » (p. I5) et inscrivent leur propos dans un "nouvel humanisme» (p. i5). Avec le Singe de l'autre, c'est finalement la question de l'altérité qui est abordée, une altérité plurielle, fondamentalement complexe mais également atteignable, une altérité qu'il est possible d'“approcher» mais aussi de “ comprendre » (p. i5).

Le Singe de l'autre est composé de deux parties. La première est consacrée à l'étude des systèmes élaborés par Lafitau et Bernard. La seconde partie porte sur la construction des images.

Dans la première partie, les auteurs s'intéressent d'abord au système de Lafitau avant d'aborder celui de Bernard et Picart. Une fois encore, la démarche suivie est très clairement explicitée. Après une présentation biographique de chaque protagoniste, les auteurs reviennent sur la méthode utilisée et sur les enjeux propres à chaque ouvrage. Le système élaboré par Lafitau repose par exemple sur le postulat diffusionniste. En repérant des coutumes observables à la fois dans le Nouveau monde et dans l'Antiquité, le père jésuite souhaite mettre en évidence des mouvements de population à grande échelle et prouver ainsi l'origine eurasiatique des populations amérindiennes. Pour les auteurs, Lafitau postule " une religion universelle, sainte dans son origine, mais sujette à la corruption »(p. 26). Les ancêtres des "sauvages» auraient donc reçu la Révélation adamique et transmis
“ une religion viciée, mais dont le fonds, essentiellement identique à celui de toutes les autres, est cependant pur» (p. 28). Le regard du père jésuite va donc des “sauvages américains » à l'Antiquité, $\mathrm{du}$ Nouveau monde vers l'Ancien. Mieux encore, sa vision de l'Antiquité est construite à partir des observations qu'il réalise en Nouvelle-France: “L'homme antique, sous son regard, devient un autre, une sorte de sauvage lui aussi. »(p.3o) Pour les auteurs, si le point de vue de Lafitau est "théologique et universaliste ", celui de Bernard est "relativiste et sceptique» (p.54). Selon ce dernier, les différentes religions peuvent être étudiées "sur un pied d'égalité » (p.32) puisque “la source de l'idolâtrie est à chercher dans la nature humaine qui est partout la même » (p. 44).

La seconde partie de l'ouvrage, intitulée “Images en parallèle », montre la place centrale qu'occupent les images dans les livres de Bernard et de Lafitau. Après un rappel des techniques de fabrication des gravures et une présentation des graveurs, les auteurs abordent la question de la construction des images : " Figurer l'Autre, en gravure, c'est littéralement bricoler avec les images du passé » (p. 63). Ph. Borgeaud et S. Petrella s'intéressent ainsi à plusieurs thèmes iconographiques, communs aux deux ouvrages, comme la "danse de religion ». Ils tâchent également d'identifier les «images sources» (p.63) utilisées et se réfèrent notamment aux travaux des mythographes et des antiquaires. Si la démonstration est tout à fait convaincante pour les premiers, le rapport à l'antiquarisme pourrait être davantage exploité. On retrouve en effet certains des objets représentés dans L'Antiquité expliquée et représentée en figures de B. de Monfaucon, dont la publication précède de peu les ouvrages de Bernard et Lafitau (planches 43 et 54 par exemple). En définitive, les auteurs mettent en évidence des procédés de déconstruction par l'image (p. 92) de la figure de l'Amérindien, un 
“ mouvement de neutralisation du sauvage » (p. 74) Les références à l'Antiquité sont mobilisées pour « comprendre le nouveau monde; pour assimiler, interpréter, nommer et convertir ce qui surgit comme des humains non christianisés » (p. Iog).

Le livre que nous proposent $\mathrm{Ph}$. Borgeaud et S. Petrella est, au sens propre, un bel ouvrage puisqu'il est orné de 83 illustrations de très grande qualité. Le singe de l'autre, élaboré en étroite collaboration avec la bibliothèque de Genève, est en effet pensé comme une contribution à l'histoire du livre. La matérialité de l'“ objet livre» est donc mise à l'honneur puisque les auteurs commentent non seulement les textes mais placent également au cœur de leur analyse les images et les processus éditoriaux, notamment dans le cadre d'un appendice, intitulé «L'habit ne fait pas le moine». L'ouvrage comprend également un utile index des noms de personnes et on regrettera simplement l'absence d'une bibliographie finale qui viendrait compléter le riche paratexte.

\section{Anna Guedon \\ Université Toulouse-Jean Jaurès anna.guedon@gmail.com}

Roberta Casagrande-Kim, Samuel Thrope et Raquel Ukeles (éd.),

Romance and reason. Islamic

transformations of the classical past,

Princeton, Princeton University Press, 20I8, I4/4.,

35 dollars / ISBN 978069II8I844.

L'ouvrage en question est le catalogue d'une exposition sur l'héritage classique dans les arts et les sciences islamiques, qui s'est tenue au printemps 2018 à l'université de New York. On peut y suivre, au travers d'un éventail de manuscrits richement colorés, les prolongements et les résonances promises dans l'Islam médiéval à des œuvres, thèmes et figures classiques telles qu'Iskandar (Alexandre le Grand). Plusieurs contributions analysent quelques facettes de cette réappropriation culturelle. On y découvre les efforts d'érudits en quête de manuscrits antiques dans les grandes villes du califat abbasside, pour les traduire, les comprendre, les commenter. De belles reproductions en couleur illustrent tout le soin que les savants juifs, musulmans ou chrétiens ont apporté à ces précieux manuscrits. L'héritage classique a fécondé l'histoire de l'Islam et la maison de la sagesse d'Haroun Al-Rachid apparaît souvent comme le symbole mémorable de cette entreprise de transmission des savoirs. Une entreprise qui s'est estompée vers le $\mathrm{x}^{\mathrm{e}}$ siècle, lorsque les savants, jusqu'à Al-Farabi, Avicenne ou Averroès ont à leur tour constitué une somme de nouveaux savoirs.

Le premier thème, mis en lumière par Julia Rubinovitch, est celui de la tradition du roman d'Alexandre. La compilation du pseudo-Callisthène $\mathrm{au} \mathrm{III}^{\mathrm{e}}$ siècle a nourri des traductions et des réécritures islamiques, qui ajoutèrent et prolongèrent tel ou tel épisode. Une mention du Coran a facilité l'islamisation de la figure grecque : l'étrange mention d'un “bi-cornu » (Dhûl-Qarnayn), envoyé par Dieu, serait une référence à Alexandre. La tradition perse a volontiers relié le conquérant à la dynastie des Achéménides, en ce qui concerne la filiation, et elle a permis grâce à la renommée de ses écoles de peinture que les manuscrits soient embellis de splendides miniatures. Rachel Milstein analyse ensuite l'iconographie d'Iskandar jusqu'à l'époque des sultanats safavide, ottoman et moghol. Les illustrations font appel à une symbolique élargie pour représenter en particulier les déterminants de la royauté et de sa légitimité.

Le deuxième thème, exploré par Leigh Chipman, est celui de la médecine. C'est un domaine dans lequel le legs de la Grèce ancienne a été précieux. Plus que le corpus 
hippocratique, c'est Galien qui a servi de référence, au moins jusqu'à l'époque des ouvrages de Razès puis d'Avicenne. Euvre majeure dans l'histoire de la médecine, le Canon de médecine de ce dernier fait usage du système des humeurs pour rendre compte des dispositions pathologiques. De nombreuses illustrations sont aussi à découvrir au fil des pages, comme celles enrichissant les traductions du traité de Dioscoride sur les plantes médicinales. Les peintures et planches ornent les traités qu'il soit question de pharmacie, de chirurgie, ou encore d'ophtalmologie, qui fut dans le monde arabo-musulman une spécialisation à part entière. Avant d'être traduite en latin aux $\mathrm{XII}^{\mathrm{e}}$-XIII ${ }^{\mathrm{e}}$ siècles, une part du savoir médical grec a bien été revue et complétée par les savants du monde arabo-musulman, d'où ce foisonnement.

Au sujet des mathématiques, de l'astronomie et de l'astrologie, Y. Tzivi Langerman revient sur l'intense activité de traduction soutenue par les premiers califes abbassides. Au cours de son développement, la science islamique a conjugué la connaissance grecque avec des apports indiens et babyloniens pour en prolonger les résultats. Les deux autorités classiques en la matière, Ptolémée en astronomie et Euclide en géométrie, ont suscité quantité de réécritures et de commentaires, de Bagdad à Samarcande. Steven Harvey aborde le dernier sujet, celui de la philosophie grecque en terre d'Islam. Le dialogue entre la falsafa (philosophie) et la religion n'a pas attiré que des regards bienveillants. Certains, comme Averroès, ont pu revendiquer le fait que l'étude et la réflexion sur les "choses existantes» ne procédait d'aucune impiété. D'autres, comme $\mathrm{Al}$-Ghazali, ont volontiers adhéré à la logique aristotélicienne, sans pour autant approuver sa conception métaphysique. Là encore les transformations islamiques de l'héritage classique, Platon et Aristote en tête, ont été nombreuses et profondes. Tandis qu'Al-Farabi demeure celui qui a exhumé la philosophie aristotélicienne et les enseignements politiques de Platon, Avicenne a mené sa réflexion philosophique sans s'interdire de désavouer parfois Aristote.

L'ouvrage offre ainsi une riche entrée en matière sur cette question passionnante de l'héritage culturel, avec de nombreuses illustrations, mais il présente aussi un panorama assez détaillé où l'on trouvera des références pour prolonger la lecture. Quelques contributions semblent parfois perdre de leur unité en déployant l'argument un peu au fil des illustrations à commenter. Néanmoins, il s'agit d'un catalogue d'exposition ayant le grand mérite de réunir des analyses et des illustrations de grande qualité, qui sont de réels outils de travail. Enfin, ce volume de belle facture est complété par le catalogue des œuvres de l'exposition et par une bibliographie.

$$
\begin{array}{r}
\text { Clément Bertau-Courbières } \\
\text { Université Toulouse-Jean Jaurès } \\
\text { clement.bertau@gmail.com }
\end{array}
$$
Hinnerk BRuHns,
Max Webers historische Sozialökonomie.
L'économie de Max Weber entre histoire
et sociologie, Wiesbaden, Harrassowitz
Verlag, 20I4, XXVI + 272 p.,
54 euros/IsBn 978-3-447-Io3og-I.

L'importance de Max Weber pour les historiens, singulièrement pour ceux de l'Antiquité, n'est plus à démontrer. Après une assez longue période d'oubli dans la première moitié $\mathrm{du} \mathrm{xx}^{\mathrm{e}}$ siècle, ses textes ont fait l'objet de lectures toujours plus attentives. En France, Hinnerk Bruhns a joué un rôle majeur dans la redécouverte de la richesse de l'œuvre de M. Weber à partir des années ig8o. Cet ouvrage en porte témoignage en réunissant des articles parus entre 1985 et 2014: treize en tout, cinq en français, huit en allemand. Cet ensemble 
offre dela sorte un utile Überblick destravaux de H. Bruhns sur M. Weber ainsi qu'une très bonne introduction aux problématiques wébériennes, particulièrement dans leur dimension historico-économique. On soulignera cependant qu'il ne comporte pas tous les travaux consacrés par H. Bruhns à M. Weber. À titre d'exemple, son texte sur M. Weber et M.I. Rostovtzeff, publié en 2005 dans Anabases, n'est pas repris. Les raisons exactes de ces choix auraient pu être mieux précisées.

Le volume est précédé de deux avantpropos (un en allemand, un en français) et d'une introduction intercalée entre le Vorwort et l'avant-propos (lequel résume en fait l'introduction en allemand). Dans cette introduction, l'auteur justifie le choix du titre par sa volonté de mettre en avant la dimension fondamentalement historique de la démarche wébérienne. L'idée est aussi de rappeler que l'économie, après la mort de M. Weber, s'est considérablement transformée en tant que discipline, l'effaçant pour longtemps de sa mémoire. En revanche, de nombreuses autres disciplines ont fait de lui un de leurs pères fondateurs, sans toujours faire l'effort de remettre ses textes et son travail dans leur contexte historique. Or c'est évidemment un des apports majeurs de H. Bruhns, historien de l'Antiquité de formation ${ }^{1}$, que de s'être intéressé à l'œuvre de M. Weber en historien, sensible à sa dimension de développement historique. Au fil de ces différents articles, il s'est agi pour lui de construire une entrée pragmatique dans l'œuvre wébérienne, avec une forte dimension contextualisante et une volonté de lecture minutieuse. Une phrase

1 Élève de Christian Meier, sa thèse, intitulée Caesar und die römische Oberschicht in den Jahren 49-44 v. Chr. Untersuchungen zur Herrschaftsetablierung im Bürgerkrieg, fut publiée en 1978 par Vandenhoeck \& Ruprecht. de la p.XIV le résume très bien: “ Im Gegenteil, es geht ganz einfach darum, Webers Beschäftigung mit bestimmten historischen Konfigurationen ernst zu nehmen, sie in Beziehung zu den großen Fragestellungen seines Werks zu setzen und sie damit auch (nicht: nur) aus seiner Perspektive als Ökonom zu lesen », ou, p. I43 : “(Re)lire Weber demande donc un effort de contextualisation et de mise en perspective historique ».

L'ouvrage est construit en trois parties. La première - “Die Stadt» - porte sur l'analyse wébérienne de la ville. H. Bruhns y revient sur ce texte posthume de M. Weber, publié en I92I, en présentant l'histoire de ce livre ainsi qu'une analyse de l'œuvre et de sa méthode. Le thème de la ville traverse en fait toute l'œuvre de M. Weber ou presque et H. Bruhns rappelle combien il est d'importance dès les Agrarverhältnisse. On retrouve là l'article le plus ancien du recueil, qui touche à la notion de ville de consommation chez M. Weber et M. I. Finley, article qui avait démontré que $\mathrm{M}$. Weber n'a pas pensé la ville de consommation telle que la présente M. I. Finley. C'est important car M. Weber fut aussi un enjeu du débat lancé par M. I. Finley sur la nature de l'économie antique. Enfin, H. Bruhns livre une présentation détaillée de la façon dont M. Weber conçoit la ville antique ainsi que les rapports familiaux qui y prévalent, ce qui donne lieu à de très intéressantes pages relatives à ces questions dans la Grèce et la Rome antiques (p. 68-84).

La deuxième partie - "Geschichte und Ökonomie» - porte sur l'économie wébérienne et sur le rapport entre histoire et économie. H. Bruhns y montre l'importance de la dimension historicoéconomique chez M. Weber (H. Bruhns parle de wirtschaftsgeschichtliche ou wirtschaftshistorische Dimension, p. 89). Il revient en particulier sur l'erreur qui consiste à couper la carrière de $\mathrm{M}$. Weber entre une première partie historico- 
empirique et une seconde (après I9oo) théorico-sociologique, erreur qui revient à minimiser toute la dimension empirique et historique de l'œuvre ultérieure ${ }^{2}$. Parce que théories et concepts sont des outils et non le but de la science, M. Weber n'a jamais abandonné la dimension empirique et historique (p. Iog et p. I77-I78). Ces chapitres resituent ainsi l'œuvre de M. Weber dans les débats économiques de leur temps (et dans la Historische Schule der deutschen Nationalökonomie, sur laquelle s'étend en détail le chapitre II.3) tout en revenant sur son rapport à la théorie économique et sur sa position (souvent mal comprise ou ignorée) dans les débats sur la nature de l'économie antique.

La troisième partie - “Weberlektüren, Weberleser» - porte sur la réception et l'influence de M.Weber chez d'autres auteurs, en particulier des historiens. Après un passionnant chapitre III.1 sur l'articulation entre science et politique au quotidien chez M. Weber, sont successivement étudiées les lectures de M. Weber par Christian Meier, Wilhelm Hennis et Fernand Braudel. De ce point de vue, le chapitre II.5, sur Otto Hinze, touche déjà à cette thématique réceptionniste en montrant les rapports entretenus par cet historien avec W. Sombart et M. Weber quant à la question du capitalisme. On soulignera au passage, dans ce chapitre, le fait qu'H. Bruhns explique l'usage fréquent des guillemets pour le mot Geist dans les textes de M. Weber (notamment ceux sur l'éthique protestante) par une référence à W. Sombart (p. I84). Ce pourrait être aussi, en réalité, une volonté “ de se démarquer

2 Cf. aussi, p. 121 la critique d'une “ approche psychologique » qui aurait conduit à surestimer la coupure de 1900, ou des remarques similaires p. 156, p. $169-170$ ou p. $191-192$. de toute référence possible au Geist de la philosophie allemande ${ }^{3}$.

À sa manière, ce livre raconte la façon dont l'intérêt pour M. Weber a considérablement augmenté en France ces dernières décennies, s'accompagnant de la parution de nouvelles et meilleures traductions, ainsi que le rôle joué par H. Bruhns dans ces évolutions. C'est aussi un témoignage de ce que l'œuvre de M. Weber n'est plus un enjeu (même si elle a pu être utilisée par le passé pour des entreprises de légitimation). Elle est à juste titre considérée comme une œuvre classique, et le débat se focalise désormais surtout sur certains concepts wébériens : comment les comprendre, comment les traduire, comment les utiliser? Cette réédition offre de la sorte au lecteur un concentré des idées de H. Bruhns sur ce sujet auquel il a consacré de longues années, et ce volume à deux langues donne un résumé du travail d'un historien qui a navigué entre France et Allemagne et qui, à côté de ses travaux, s'est aussi attaché à jouer un rôle de passeur entre ces deux univers académiques si proches et pourtant parfois si lointains. Les textes se répondent avec étonnamment peu de répétitions, certains développant des points rapidement traités par d'autres, ce qui confère une réelle unité à l'ensemble. On y trouve un Weber historien, un Weber empirique (cf. les rappels sur l'usage de l'idéaltype), un Weber réinséré dans les débats de son temps. S'y déploie aussi une lecture attentive et patiente des textes de M. Weber, une lecture d'historien en somme.

$$
\begin{array}{r}
\text { Thibaud Lanfranchi } \\
\text { Université Toulouse-Jean Jaurès } \\
\text { lanfranc@univ-tlse2.fr }
\end{array}
$$

3 Cf. J.-P. Grossein, “Présentation », dans M. Weber, L'Éthique protestante et l'esprit du capitalisme, suivi d'autres essais, Paris, Gallimard, 2003, p. XLI n. 2. 


\author{
Andrea Cozzo, \\ Riso e sorriso, e altre saggi sulla \\ nonviolenza nella Grecia antica, \\ Sesto San Giovanni (Milano), \\ Mimesis Edizioni, \\ Collana Classici contro, n. II, 20I8, \\ ı6 euros /IsBn 885755 ı 873 .
}

L'ouvrage ici recensé est un recueil de six essais, dont les deux premiers sont inédits, alors que les suivants (3-6) sont des versions enrichies de textes déjà publiés (cf. note 2). Une introduction explique le sens de cette entreprise. Le lien entre les différentes contributions est tout d'abord thématique car toutes trouvent leur cadre dans un programme de recherche sur la Grèce ancienne qui a pour objet les pratiques de gestion des conflits excluant le recours à la violence, y compris celle de défense. Andrea Cozzo veut écrire une histoire démilitarisée, qui raconte les processus de construction de la paix : à cette fin, il met en lumière ce qui est généralement négligé par les historiens, à savoir les pratiques et les formes de pensée alternatives à la logique militaire. Outre les thèmes, le lien entre les différents essais est donné par les objectifs de l'auteur, qui ne relèvent pas uniquement de la science: comme il le revendique dans son introduction, il s'agit d'un travail politiquement engagé, qui veut utiliser le passé pour participer à l'élaboration et à la diffusion d'une culture de la paix et contribuer ainsi au développement d'une société moins violente. De façon cohérente avec ce projet, le livre se destine à un public plus large que celui des spécialistes de l'Antiquité : le langage est peu technique, le ton narratif. Les différents essais retrouvent dans le monde grec les traces des idées fondamentales de la non-violence gandhienne: les trois premiers, sur un plan interpersonnel, les trois suivants sur un plan plus large, social ou interétatique. Par ailleurs, ce volume se situe dans la continuité avec un autre travail, " $\mathrm{Nel}$ mezzo ». Microfisica della mediazione nel mondo greco antico, Pisa, 2014, dans lequel l'auteur avait déjà très largement étudié les pratiques d'arbitrage, de médiation et d'interpositions dans la Grèce ancienne, d'Homère au v viècle ap. J.-C. Le premier essai, Riso e sorriso nella trasformazione dei conflitti, examine le rôle reconnu dans la culture grecque au rire et au sourire dans la gestion non violente des conflits : si le rire est en général agressif, provocateur, et devient rapidement dérision, le sourire est en revanche un geste non invasif, non excessif, permettant d'enclencher un retour en arrière de la violence. Le deuxième essai, Suggerimenti filosofici su come gestire $i$ conflitti interpersonali nella vita quotidiana, synthétise l'enseignement de plusieurs philosophes anciens à propos des différentes étapes dans la gestion d'un conflit interpersonnel. Le troisième essai, Del potere (nonviolento) che spesso si ignora di avere, retrace les critiques grecques à l'idée de la nécessité d'une réponse violente à la violence et reconstruit les règles (grecques) pour une réaction non-violente à la prévarication subie: l'auteur présente de nombreux exemples de non-collaboration avec l'oppresseur ou de désobéissance civile. Le quatrième chapitre, Donne operatrici di pace nel mondo greco (e non solo), identifie une modalité propre à l'action des femmes en cas de conflits, qui ne coïnciderait pas avec un refus faible de la violence militaire, mais qui ne correspond pas plus à une participation virile à la guerre: les femmes, épouses et mères de guerriers, ont une place particulière, “ au milieu »; leur action se concrétise dans des pratiques de médiation et d'interposition entre les parties. Le cinquième chapitre, Come evitare le guerre e rendere amici $i$ nemici. Forme della diplomazia nella Grecia Antica, rappelle les actions diplomatiques, qui permettent la reprise des relations après une fracture, avec ou sans l'intervention de tierces personnes en tant qu'arbitres ou médiateurs. Un dernier chapitre, Dialogare tra $i$ nemici, part du constat que ce qui 
permet à un homme de tuer un autre homme sans ressentir cela comme un problème éthique est la distance qui les sépare. Ainsi, Cozzo retrouve les moyens grecs de réduire cette distance pour permettre un dialogue entre les parties en conflits. Le livre d'Andrea Cozzo est atypique : une médiation réussie entre érudition, vulgarisation et écriture historiographique. Il s'agit d'une œuvre érudite : Cozzo se sert d'un nombre impressionnant d'auteurs et de textes pour démontrer le postulat posé, à savoir que les Grecs ont aussi théorisé autre chose que la violence; mais c'est également un livre de vulgarisation, qui peut être lu avec plaisir et intérêt par tout public : l'auteur raconte des dizaines d'anecdotes et de petites histoires pour créer des tableaux et rendre vivantes les conclusions auxquelles il veut parvenir ; enfin, il s'agit d'un livre historiographique, dans la mesure où l'auteur se positionne dans un débat (comment écrire l'histoire?) pour refuser l'idée de l'histoire comme un enchaînement de guerres interrompues par des moments de paix. Cozzo cherche à ancrer une culture de paix dans notre passé européen commun et se présente explicitement comme le plus récent maillon d'une tradition ancienne de non-violence qui remonte (au moins) à la Grèce ancienne. L'auteur utilise de manière explicite les textes anciens pour créer un manuel contemporain du citoyen non-violent. En plus de renseigner sur les anciens, ce livre donne envie d'être meilleur. On pardonnera à l'auteur avec un sourire bienveillant une attaque gratuite contre les philologues et leurs bavardages (p. 4): tous frais de sa lecture, on ne ripostera pas, certains qu'il regrette cette inélégance.

Francesca Prometea BARone IRHT, CNRs, Paris francesca.barone@irht.cnrs.fr
Franz Cumont, Manichéisme, éd. D. Praet et M. Tardieu, avec la collaboration d'A. Lannoy et d'A. Di Rienzo,

Rome, Nino Aragno Editore (diffusion Brepols, Turnhout), 20I7, XLIII et 209 p., 75 euros / ISBN 978-90-7446I-87-0.

L'entreprise de réédition des œuvres de Franz Cumont dans la magnifique « Bibliotheca Cumontiana» se poursuit par les Scripta Minora, série qui comptera à terme sept volumes et dont voici le premier qui réunit toutes les publications de F. Cumont sur le manichéisme. Les dix-sept études réunies ici sont originellement parues de 1907 à 1938 et sont d'importance et de volume variés. Elles manifestent cependant toutes le vif intérêt que F. Cumont n'a cessé de porter toute sa vie à la religion, à la personne et aux écrits de Mani. L'historien des religions qu'il était avant tout pensa trouver dans la philosophie du “Paraclet » (Mani, qui connaissait les Évangiles, se présentait lui-même ainsi ou encore comme “l'apôtre de Jésus-Christ»; voir p. I76) une mutation du mithriacisme et c'est l'espoir que lui inspirait cette idée de mieux comprendre le culte de Mithra qui poussa F. Cumont à entreprendre, en Igoo, son grand voyage à Trébizonde. Ainsi que le dit M. Tardieu, "s'il n'y rencontra pas Mithra, il y trouva Mani » (p. XXII).

Parmi les résultats de ces dix-sept études on retiendra notamment cette correction palmaire du texte du Contra Faustum (I5, 6) d'Augustin dans lequel F. Cumont proposa le premier de voir en adamantem (p. 428 Zycha, CSEL 25 [I], I89i) non pas un adjectif épithète de heroam (“ un héros de diamant ") mais le nom propre Adamas, emprunté aux gnostiques, désignant ce personnage chanté par les manichéens pour qui il était “l'Homme céleste, être androgyne, premier principe de toutes choses» (p. 7I). Dans son étude sur «La propagation du manichéisme dans l'Empire 
romain »(p. 73-8I) F. Cumont affiche son goût pour les longues perspectives et les larges visions. L'article fait l'histoire du manichéisme "sorti de Babylonie vers l'an 275 de notre ère ", date de l'exécution de Mani par le roi de Perse Bahram I ${ }^{\text {er }}$ qui le fit écorcher à l'époque du règne de Probus (on connaît le sort identique de l'empereur romain Valérien supplicié par les Perses, aux dires de l'Histoire Auguste, après sa capture à Édesse en 26o). Mais F. Cumont suit encore les traces de la religion de Mani aux III $^{\mathrm{e}}$ et $\mathrm{IV}^{\mathrm{e}}$ siècles dans “ l'Orient sémitique », en Asie Mineure, en Égypte et bien sûr jusqu'à Rome où, on le sait, saint Augustin débarqua en 383 avant de trouver refuge dans les milieux manichéens actifs de la cité où ils vivaient leurs croyances clandestinement. Enfin, même si aujourd'hui on ne suit plus F. Cumont sur ce plan, on notera que pour ce dernier le manichéisme, qui aurait connu Marcion et la gnose, a survécu dans l'hérésie de Priscillien et, plus tard, dans le catharisme. Dans la conclusion d'une étude puissante par sa force évocatrice F. Cumont définit à merveille le dualisme manichéen " qui opposait partout la matière perverse à l'esprit divin » et explique comment les adeptes de Mani pratiquèrent un ascétisme intransigeant (voir l'inscription funéraire de Salone publiée par F. Cumont, p. I47, qui donne le nom d'une « vierge » manichéenne nommée Bassa et originaire de Lydie). Il faut lire ensuite la longue et riche édition que F. Cumont avait proposée avec M.-A. Kugener (I873-I94I) (le premier commente, le second traduit) d'un texte de Mani ( Extrait de la CXXIII ${ }^{e}$ homélie de Sévère d'Antioche », p. 83-i45). Ce dernier, on le sait, écrivait en syriaque. Or le patriarche d'Antioche (5ı2-5ı8) Sévère a conservé dans son homélie écrite initialement en grec de très longs passages d'un texte de Mani que F. Cumont identifie avec Le Livre (ou Traité) des Géants. Mais de l'homélie de Sévère nous n'avons plus qu'une traduction syriaque : le texte original de Mani a donc subi deux traductions, du syriaque d'abord vers le grec, puis du grec de Sévère vers le syriaque, avant d'être traduit et commenté par M.-A. Kugener et F. Cumont. Dans ce texte l'auteur développe son dualisme fondamental sous la forme d'une parabole, celle de la lutte entre l'Arbre de la Vie et l'Arbre de la Mort. Plusieurs notes ou lettres envoyées par F. Cumont à des revues attestent qu'il a constamment été attentif aux « trouvailles » (comme il aimait à dire) de manuscrits supposés contenir des textes manichéens, par exemple ces livres rédigés dans un dialecte copte de la Haute-Égypte contenant notamment un "Capitulaire " de pas moins de 520 pages (p. I78) dont il mentionne en ig33 la découverte dans la Revue de l'Histoire des Religions (ici p. I77-I83).

Mais la plus grande richesse de cette réédition des articles sur le manichéisme de F. Cumont réside dans l'introduction historiographique d'usage dans la collection et ici rédigée de main de maître par Michel Tardieu. Ce dernier donne une liste précise et commentée des livres et des auteurs qui ont influencé F. Cumont ou qui ont, plus tard, collaboré à ses travaux de commentaire en lui fournissant des traductions de l'arabe ou du syriaque. On y retrouve notamment le nom du professeur strasbourgeois Theodor Nöldeke (I836I930) ou celui de Konrad Kessler (I85I-I9o5). M. Tardieu analyse encore comment Adolf von Harnack reçut plutôt positivement les travaux de F. Cumont sur le manichéisme tout en soulignant les limites des rapprochements chers à l'auteur entre le mithriacisme et le christianisme. Cette riche et érudite introduction envisage enfin d'un point de vue historiographique les différentes explications qui ont pu être proposées à l'échec historique du manichéisme. Si F. Cumont considérait que l'État romain par ses interdictions successives (à commencer par l'édit de Dioclétien en 297) fut le principal vainqueur, d'autres auteurs, rappelle M. Tardieu, penchent plutôt, comme Louis Massignon, pour le refus d'une reli- 
gion unique et dominatrice ou encore, comme Walter Burkert (I93I-20I5), mettent en avant l'ascétisme radical des disciples de Mani. M. Tardieu offre enfin une «Mise à jour » bibliographique et critique (p. XXXV$\mathrm{XL}$ ), numéro par numéro, des dix-sept études de F. Cumont. Ce travail aurait justifié à lui seul, tant il est précieux, l'existence de la réédition des études de F. Cumont. Mais le philologue se plongera avec le plus grand profit dans les traductions des textes manichéens que F. Cumont sait commenter avec une science et dans une langue qui n'appartiennent qu'à lui.

Stéphane Ratri Université de Bourgogne Franche-Comté stephane.ratti@univ-fcomte.fr

Emmanuelle HÉnin et Valérie NAAs (dir.), Le mythe de l'art antique, Paris, CNRs Éditions, 20I8, 484p, 25 euros / ISBN 978-2-27I-0902O-I.

Le recours à la figure de l'artiste antique est devenu un véritable topos dans la littérature artistique, des vies d'artistes aux textes normatifs, en passant par les œuvres elles-mêmes, que l'on pense au portrait ironique de Rembrandt en Zeuxis ou aux représentations d'Apelle au Cinquecento. C'est cette utilisation polysémique des récits mettant en scène des artistes antiques qui fait l'objet d'un ouvrage essentiel, permettant à la fois une mise au point historiographique et conceptuelle sur le sujet et l'ouverture de nouvelles perspectives. Sous la direction d'Emmanuelle Hénin et de Valérie Naas, Le mythe de l'art antique est introduit par une préface d'Agnès Rouveret qui annonce les cinq axes structurant l'ensemble et les questions qu'il suscite, à savoir celles des fonctions et modalités d'usage des anecdotes sur l'art antique, de sa consti- tution en mythe et son évolution selon les types d'œuvres et les époques.

C'est ainsi que la première partie, “ Cadres rhétoriques et épistémologiques », établit les fondations nécessaires à la construction notionnelle du livre. Le premier essai d'E. Hénin problématise la question en présentant la base de données Pictor in fabula $^{4}$ suivant une typologie croisant fort à propos théorie du ut pictura poesis et interprétation génétique pour décrypter ce vaste palimpseste des récits sur la peinture antique. P. Duarte poursuit cette réflexion sur l'intertextualité à travers son analyse typologique et syntaxique des discours fictifs dans les anecdotes artistiques. Les deux contributions suivantes, consacrées respectivement à Timanthe (Pline, Histoire naturelle, XXXV, 73) par G. Lombardo et à Jean Tzetzès par S. Kuttner-Homs, analysent les rapports de ces anecdotes à la rhétorique : le premier met en avant un système d'équivalences des éloquences visuelle et textuelle, tandis que le second souligne comment les anecdotes peuvent servir les desseins littéraires des auteurs qui les emploient. La dernière étude, de M. Delbeke, clôt remarquablement la partie en mettant en perspective les fonctions épistémologiques de ces histoires et leur malléabilité pour illustrer un propos théorique plus large, qu'il soit d'ordre philosophique, psychologique ou sociopolitique.

La deuxième partie, “Anecdotes et pratiques de la peinture » interroge le rapport de ces textes antiques aux pratiques artistiques effectives et à leurs significations. Ainsi V. Naas éclaire-t-elle la construction de la peintre femme renaissante à partir de la relecture des anecdotes de Pline par Boccace. L'anecdote du bœuf de Pausias (Pline, Histoire naturelle, XXXV, I26I27) analysée par B. Saint Girons permet d'envisager la question problématique de la

4 http:/http://www.pictorinfabula.com. 
skiagraphie. R. Dekoninck s'attache, quant à lui, à souligner les rapports d'aemulatio à l'œuvre entre art et nature par l'utilisation polysémique de l'histoire de la rivalité amoureuse entre Pausias et Glycère (Pline, Histoire naturelle, XXXV, I25) dans l'œuvre de Rubens: l'anecdote plinienne devient alors support méta-iconique par une mise en abîme des références qu'elle véhicule. Cette même histoire est reprise par M.-P. Martin qui propose une réflexion sur la dimension scientifique du récit mise en relation avec la question de l'harmonie des couleurs chez Newton. É. Jollet évoque ensuite le concours de la ligne d'Apelle et Protogène (Pline, Histoire naturelle, XXXV, 8I-83) afin d'analyser la question de la durée d'exécution dans la littérature artistique française du xvIII $^{\text {e }}$ siècle et l'œuvre de Fragonard et, plus largement, la fonction mnémonique de l'anecdote. Les deux dernières contributions prennent pour point de départ un exemple pictural, Zeuxis peint le portrait d'Hélène de Domenico Beccafumi pour É. de Halleux, et le Sacrifice d'Iphigénie de Jan Steen pour J. Blanc, pour développer une réflexion sur la théorie de l'art. La première propose ainsi une étude éclairante de l'idée de beauté recomposée et unifiée dans la littérature artistique du $\mathrm{XvI}^{\mathrm{e}}$ siècle, tandis que le second s'appuie sur un commentaire du tableau de Steen par Joshua Reynolds pour caractériser le burlesque des anecdotes antiques au service d'une “ contre-poétique du ridicule »(p. Ig3), le traitement du voile de Timanthe cachant le visage d'Agamemnon devenant un moyen d'affirmation de l'esthétique de l'artiste.

Cela amène à un glissement du champ épistémologique du traitement de l'anecdote antique à celui du topos qui en découle dans la troisième partie, "Topoi mimétiques ». Celle-ci commence par une étude de G. Pucci sur la rivalité entre Zeuxis et Parrhasios (Pline, Histoire naturelle, XXXV, 65-66), de laquelle découle un poncif de la peinture: sa quête d'illusionnisme et le recours au trompe-l'œil. Le rideau peint par Parrhasios devient la métaphore de cette recherche picturale questionnant le rapport du spectateur à l'œuvre. F. IffNoël définit ensuite le caractère mimétique du topos à travers une analyse précise de la typologie et des structures des œuvres si réalistes qu'elles semblent sur le point de parler au service d'une réflexion plus large sur le ut pictura poesis : la parole impossible de l'œuvre d'art signifie in fine la supériorité de la littérature qui «donne la parole à l'art pour mieux révéler son mutisme» (p. 223). La contribution de J. Riello envisage l'apport des anecdotes antiques dans la réflexion artistique hispanique du $\mathrm{xvII}^{\mathrm{e}}$ siècle, les théoriciens s'appuyant sur les exemples antiques pour affirmer la nécessité de l'imitation du naturel si caractéristique des tableaux du Siècle d'Or, tandis que L. Pouy les étudie dans leur rapport aux œuvres de Gerrit Dou et propose ainsi une nouvelle lecture de son célèbre tableau du Louvre, la Trompette. Après une contribution de P. Butti de Lima sur la question de la mise en images de la douleur, l'essai de F. Lecercle clôt avec brio cette partie en montrant comment par la littérature artistique Michel-Ange est devenu l'émule moderne de Parrhasios, deux artistes « tortionnaires ».

La quatrième partie plus courte, "La mimesis sculpturale », permet d'approfondir les problématiques déjà présentées dans le champ spécifique de la sculpture. R. Robert offre ainsi une contribution indispensable sur la figure de Phidias, les questions de son statut social et de l'évolution de son image au fil de l'Antiquité. M. Cojannot-Le Blanc et É. Prioux étudient ensuite les significations du recours aux sculpteurs antiques dans les épigrammes sur le buste de Louis XIV du Bernin, tentative de recréation du couple formé par Alexandre et Apelle. M. Brock s'appuie quant à lui sur quatre lettres d'humanistes italiens (Bembo, l'Arétin, Della Casa et Dolce) pour souligner comment l'anecdote plinienne de l'Aphrodite de Cnide de Praxitèle (Pline, 
Histoire naturelle, XXXVI, 20) sert un propos visant à affirmer le principe de délectation comme finalité des œuvres profanes, telles les œuvres mythologiques de Titien. Cette même anecdote relevant du registre comique permet enfin à L. Wajeman d'envisager sa relecture sur un mode sérieux à la Renaissance, au service de l'affirmation de la dignité de l'art.

La cinquième partie, "Portraits de l'artiste en Apelle », offre enfin un focus sur la figure tutélaire du peintre d'Alexandre. M. Bert analyse avec précision la question du choix de signer à l'imparfait de Fra Damiano ses décors en bois de l'église San Domenico de Bologne pour signifier implicitement son émulation avec Apelle et revendiquer la marqueterie comme art à part entière. S. Blake McHam étudie quant à elle le recours au topos de l'artiste amoureux de son modèle, notamment la fille du potier Butadès (Pline, Histoire naturelle, XXXV, I5I-152), Apelle et Campaspe (Pline, Histoire naturelle, XXXV, 86-87) et Praxitèle et Phryné, par les théoriciens et peintres de la Renaissance italienne comme mise en abîme de la recherche du beau idéal. La contribution d'A. Fabbricatore permet d'élargir la réflexion au domaine de la danse par l'analyse de la figure d'Apelle dans le ballet d'Apelle et Campaspe de JeanGeorges Noverre, alors que F. R. Martin ouvre chronologiquement le sujet sur l'usage de l'anecdote d'Apelle et Campaspe puis de celle de Raphaël et de la Fornasina dans l'œuvre d'Ingres signifiant que l'artiste devient au début du XIX ${ }^{\mathrm{e}}$ siècle son propre souverain " dans une érotique de la création qui laisse le peintre seul avec son modèle » (p. 442).

C'est donc un ouvrage essentiel qui nous est ici proposé par l'intérêt et la diversitétant chronologique, géographique, artistique et littéraire des champs de recherche abordés et des exemples analysés et l'intérêt des perspectives dégagées, qui font de ces anecdotes antiques des textes fondateurs, sources indispensables pour interroger la littérature artistique et l'histoire de l'art modernes, en particulier pour examiner les notions de paragone des arts, d'imitation et d'émulation. Toutes les références antiques sont rassemblées en fin d'ouvrage avec un index (p. 459-484), ce qui facilite la lecture et la circulation dans l'ensemble du livre. L'ouvrage en décortiquant les processus artistiques à l'œuvre dans le recours au topos antique ouvre ainsi la voie à de nouvelles approches et questionnements, recherches récemment poursuivies autour de la figure de Zeuxis et de la problématique de l'émulation lors d'un colloque organisé par M. Bert, R. Dekoninck et L. Fagnart ${ }^{5}$.

Clarisse Evrard

École du Louvre clarisse.evrard@wanadoo.fr

Jacques Jouanna, Henri Lavagne, Alain PASQuier, Véronique SchiLtz et Michel Zink (éd.), Au-delà du Savoir : Les Reinach et le Monde des Arts,

Paris, Diffusion De Boccard, 20I7, 3oo p., 3o euros/ IsBN 978-2-87754-36o-6.

Édité par Jacques Jouanna, Henri Lavagne, Alain Pasquier, Véronique Schiltz et Michel Zink, cet ouvrage est la publication du $27^{\mathrm{e}}$ colloque qui s'est tenu, les 7 et 8 octobre 20ı6, à la Villa Kérylos, sous la présidence de Michel Zink. Outre l'allocution d'accueil et la conclusion, il compte I2 contributions. Le précédent colloque, organisé en 2006 et publié en 2008, avait présenté la fratrie d'un point de vue intellectuel, scientifique et politique. Juifs d'ascendance allemande, français,

5 Zeuxis redivivus. Art et émulation dans l'Europe du XIV au XVII siècle, colloque international, Bruxelles, Palais des Académies, 27-29 septembre 2018. 
laïcs et républicains, les frères Reinach, Joseph (I856-I92I), Salomon (I858-I932) et Théodore (I860-I928), étaient surnommés les «Frères Je-Sais-Tout » en raison de leur prodigieuse érudition. Dans son allocution d'accueil (p. I-VII), Michel Zink relève que l'objectif du colloque de 2or6, placé sous le patronage de l'Académie des Inscriptions et Belles-Lettres, était d'aller « au-delà du savoir » en mettant au jour les incursions des frères Reinach dans la modernité. Cette enquête est élargie à deux héritiers Adolphe (ı887-19ı4), fils de Joseph, et Léon Reinach (I893-I944), fils de Théodore, en raison de leur intérêt pour la peinture et la musique.

Par son étude sur les Reinach et les revues savantes (p. I-I8), Jacques Jouanna cherche à comprendre comment « le savant solitaire dans son bureau se transforme en homme du monde, ou du moins en homme dans le monde » (p. I). Salomon et Théodore ont dirigé deux revues, le premier la Revue archéologique à partir de 1903, le second la Revue des Études grecques à partir de 1888. Théodore fut à l'initiative d'une chronique élargie à la Grèce byzantine et moderne, dont subsistent aujourd'hui le Bulletin céramologique et le Bulletin épigraphique. Il fut aussile directeur de la Revue de la Gazette des Beaux-Arts de I9o5 à I928, embrassant “l'étude rétrospective et contemporaine de toutes les manifestations de l'art et de la curiosité (architecture, sculpture, peinture, gravure, arts décoratifs, musique) ». Pour souligner l'apport de Théodore Reinach à la musique grecque antique (p. I9-6o), Christophe Corbier rappelle combien cet art est resté longtemps textuel et technique, relevant de la philologie et de l'esthétique, faute de documents musicaux. Théodore Reinach a poussé très loin « la rencontre de l'érudition la plus fouillée et d'un imaginaire hellénique dont la musique est un des éléments essentiels » (p. 2I). Ayant édité les deux hymnes trouvés à Delphes en I893, il a écrit les livrets de La naissance de la lyre et de Salamine, le premier à partir de l'Hymne homérique à Hermès et des
Limiers de Sophocle, le second à partir des Perses d'Eschyle. Fauré fut chargé par Reinach d'adapter le premier hymne delphique au "sentiment musical actuel » (Hymne à Apollon opus 63 bis). Comme son interprétation ne manqua pas de susciter de vives critiques, Reinach se défendit d'avoir cherché à restituer l'hymne tel qu'il était. Il avait cherché à l'adapter au public. La tragédie lyrique Salamine - adaptation des Perses d'Eschyle - marqua le point d'aboutissement des travaux de Reinach et de Maurice Emmanuel. Ce dernier, auteur d'une thèse sur La Danse grecque antique, partageait les convictions de Théodore Reinach: les textes ne sont pas seulement des documents archéologiques, ils doivent prendre vie pour transmettre des valeurs et permettre de régénérer la société française. Agnès Rouveret étudie les textes originaux et les commentaires rédigés par Adolphe Reinach (I887-I9I4) pour un projet de recherche sur la peinture antique dont il eut la responsabilité en I9II (p. 6I-84) et que la donation du peintre Paul Milliet (I844I9I8) avait rendu possible. L'ouvrage publié sous le nom de Recueil Milliet (réédition, I985) contenant une partie du manuscrit d'Adolphe Reinach sur les peintres, Agnès Rouveret peut établir des correspondances entre ses analyses et l'art de son temps grâce aux tableaux qui figuraient dans la famille et à la palette des couleurs du décor de la Villa Kérylos. Adolphe Reinach, “jeune érudit de la plus belle espérance », tout en concevant son projet dans un esprit d'émulation avec la science allemande, chercha à diffuser largement ses connaissances sur l'art antique. Filippo Tuena, auteur des Variations Reinach, livre édité en 2005 et réédité en 20I5, a découvert, à la bibliothèque de Harvard, la partition de la seule composition de Léon Reinach qui nous soit parvenue, une sonate pour violon et piano en ré mineur, probablement écrite pour l'obtention de son diplôme au Conservatoire de Paris, en I925. Dans sa contribution au colloque (p. 85--го), il juge 
utile de préciser, grâce à des documents jusque-là inédits, les circonstances de l'arrestation de Léon Reinach et de sa famille par les nazis. Il souligne que le destin des Reinach fut bien plus dramatique que ce que l'on croyait puisqu'ils ne furent pas supprimés parmi les premiers lors de leur arrivée à Auschwitz. Alain Pasquier propose de retrouver les noms et les visages des musiciens du conservatoire que Léon Reinach a côtoyés, en ayant recours aux photographies et aux portraits en forme de caricatures que son père instrumentiste a crayonnés (p. Io3-I28). Il s'étonne que l'œuvre musicale de Léon soit réduite à une sonate et à deux mélodies, faible activité qu'il attribue au déclin des salons de musique dans les années 20. Philippe Cathé centre sa contribution sur Charles Koechlin, compositeur prolixe (I867-I95o) et auteur du Traité de l'orchestration (p. I29-I49). Reconnu par ses maîtres Fauré et Debussy, il s'était vu confier par Théodore Reinach la critique musicale dans les deux revues qu'il dirigeait, entre Igog et I92I. Philippe Cathé souligne que les deux hommes font de la Grèce la source de l'art musical français contemporain. Antoine Compagnon évoque le «milieu parisien de la bourgeoisie éclairée de la rive droite» dans son étude : “Proust, les frères Reinach et Emmanuel Pontremoli »(p. I5I-I66). Proust, élève au lycée Condorcet comme les frères Reinach, ne pouvait qu'être impressionné par leur réussite scolaire. Antoine Compagnon analyse les sentiments de Proust pour Joseph Reinach, qu'il fait apparaître dans Le Côté de Guermantes et dans Le Temps retrouvé. En novembre i4, l'écrivain sollicita Joseph Reinach pour obtenir sa radiation de toute obligation militaire. Or, il n'obtint pas satisfaction: Joseph, ayant perdu son fils et son gendre dès le début de la guerre, le considérait comme un embusqué. Bien qu'il admirât le sérieux des Commentaires de Polybe, nom sous lequel Joseph tenait une chronique dans le Figaro, Proust tourna en dérision leur style pédant, digne du concours général, comme s'il n'avait pas oublié les années de lycée et l'agacement que provoquaient les frères Reinach pour leur excellence.

Les frères Reinach ont grandi au milieu des œuvres d'art et en particulier des tableaux de l'école italienne. La peinture leur fut une passion commune, souligne Hervé Duchêne (p. 167-I87). Salomon décida de lancer un inconnu, citoyen américain, Bernard Berenson, auteur de quatre essais sur la peinture italienne, qui devint le pape des connaisseurs dans ce domaine. De cette Église, Salomon fut “ l'évangéliste » (p. i68). Salomon, séduit par l'originalité de ses monographies, voyait en lui celui qui devait révolutionner la critique d'art. Il y a un zeste de provocation dans le titre de la longue étude que Véronique Schiltz consacre aux Reinach “ entre raison et déraison » (p. I89228), tant la fratrie s'inscrit dans la tradition du savoir encyclopédique. Elle entend prouver que loin d'être déraisonnables, les Reinach ont côtoyé la déraison. Ils ont en effet vécu dans une époque qui croyait au progrès scientifique tout en s'intéressant au paranormal. Leur ami, Charles Richet, prix Nobel de médecine, pacifiste et dreyfusard, a été fasciné par Eusapia Palladino qui faisait tourner les tables et soulevait sans la toucher une table de $20 \mathrm{~kg}$. Il lui attribuait des bras et des mains ectoplasmiques. Joseph Reinach, auteur d'un ouvrage Contre l'alcoolisme et qui mena un combat au Parlement pour la sobriété, a fait la publicité d'une boisson alcoolisée le vin Mariani, fabriqué à partir de vin de Bordeaux et d'extrait de feuilles de " coca du Pérou ». Sur une carte qui loue cette boisson, son frère Salomon déclare: «Le vin, a dit Aristophane, est le lait d'Aphrodite. Le vin Mariani est celui d'Athéna ». Salomon semble illustrer au mieux ce thème « entre raison et déraison " par ses relations avec les femmes. Il s'intéressa de près au corps féminin et particulièrement à la poitrine. Dans un mémoire, il proposa de prendre le plus ou moins grand écartement entre 
les seins comme indice de datation des korès. Auteur d'un ouvrage remarqué sur la religion qui fait preuve de rationalité, Cultes, mythes et religions, il se passionna, dès I894, pour une mystique du XviI ${ }^{\mathrm{e}}$ siècle Antoinette Bourignon, auteur d'un Traité de la solide vertu. Il fut aussi attiré par des femmes marginales. Il soutint le projet du château Les Avenières à Cruseilles que fit construire, de 1907 à I9I3, Mary Shillito dans un style néo-gothique. Sous l'influence d'Assan Dina, assyriologue féru d'ésotérisme, que Salomon lui avait présenté en juin ı9ı3, elle métamorphosa le château en demeure ésotérique, dans un esprit à mille lieues de la laïcité militante de Salomon Reinach. Non sans humour, Véronique Schiltz fait de la Villa Kérylos la plus grande déraison des trois frères. Propos qui introduit la contribution suivante: “La Villa Kérylos : le projet d'Emmanuel Pontremoli pour Théodore Reinach »par Pierre Pinon (p. 229-249). La rencontre entre Reinach et Pontremoli «était écrite dans leurs origines, dans leur goût commun pour l'Orient hellénisé » (p. 234). Théodore Reinach (I86o-ıg28) est d'abord un défenseur de la laïcité; Emmanuel Pontremoli (I865-ı556), issu d'une famille juive piémontaise pratiquante, apprend à aimer l'Antiquité et ses monuments lors de plusieurs voyages. Trois ans après avoir remporté le Grand Prix de Rome, il se rend en Grèce où il découvre les sites les plus fameux, dont celui de Delphes, où il est guidé par Théophile Homolle qui dirigeait alors les fouilles. Quand le projet de la Villa Kérylos se concrétise en face de Beaulieusur-Mer où la famille de Pontremoli avait une propriété, il s'enthousiasme pour cet emplacement à même d'évoquer la vie antique. La Villa ouvrit à Pontremoli une carrière d'architecte et d'enseignant à l'École des Beaux-Arts dont il devint directeur en 1932. Pour Michel Jarrety (p. 25I-262), rien ne semblait pouvoir rapprocher Salomon, normalien, ancien élève de l'École d'Athènes, archéologue et spécialiste de l'histoire de l'art et des religions, et la poétesse anglaise Pauline Tarn, connue sous le pseudonyme de Renée Vivien pour ses poèmes sur le saphisme. Renée Vivien étant morte en Igog à l'âge de 32 ans, Théodore Reinach ne l'a pas connue mais s'est prise pour elle d'une passion posthume grâce à la rencontre, en I9I4, de Nathalie Barney, premier grand amour de Pauline Tarn. Il travailla sur l'œuvre de Renée Vivien, archiva, commenta, rassembla des documents pour les historiens et biographes du futur, “ les historiens à lunettes qui écriront sur Pauline en 2000 ». Pour lui, Pauline Tarn est « une fille de génie et le plus grand poète du $x^{\mathrm{e}}$ siècle» (p. 253). Jean-Loup Fontana (p. 263-27o) présente l'histoire musicale de Nice et son essor dans la seconde moitié $\mathrm{du}$ хvıII $^{\mathrm{e}}$ siècle, mais les frères Reinach réservaient leur activité artistique à un cénacle à l'écart de la société niçoise. En guise de conclusion (p. 27I-274), Henri Lavagne souligne combien cette rencontre a entraîné les participants dans des domaines divers, la musique, la peinture mais aussi l'occultisme et le monde saphique.

Les trois frères n'ont pas la même place dans le colloque, que ce soit pour les thématiques développées ou pour leur ouverture sur le monde des arts. L'aîné Joseph, chef de cabinet de Gambetta et député en I889, réélu en I893, est cité à propos de ses positions dans l'affaire Dreyfus dont il se fit l'historien, il l'est également à propos de son combat contre l'alcoolisme. Les cadets Salomon et Théodore occupent la plus grande partie des contributions en raison de leur intérêt “au-delà du savoir " pour des domaines éloignés de leur spécialité. Justice est rendue à Adolphe Reinach pour sa passion pour la peinture et à Léon Reinach puisque furent jouées à la Villa Kérylos sa sonate en ré mineur pour piano et violon ainsi que des mélodies.

Le colloque a quitté les territoires de l'érudition et de l'Antiquité pour découvrir 
chez les Reinach les hommes qu'ils furent, intéressés par les nouveautés de leur siècle. Érudits, scientifiques et rationnels, ils n'en étaient pas moins passionnés par l'irrationnel et la Modernité.

Geneviève HofFmann Université de Picardie Jules Verne genevieve.hoffmann@wanadoo.fr

\author{
Mario Liverani, \\ Imagining Babylon: \\ The Modern Story of an Ancient City, \\ Berlin-Boston, De Gruyter, \\ Studies in Ancient Near Eastern \\ Records II, 20I6, xviii +488 p. \\ ı82 dollars / ISBN 978ı6ı/5ı6026.
}

Le livre de Mario Liverani est un tour de force et il embrasse une matière infiniment plus large que ce que le titre suggère. Certes, il est bien question de l'histoire moderne de la cité antique de Babylone - l'histoire de sa découverte et de ses multiples interprétations - mais plus globalement, ce que Mario Liverani offre, dans ce livre remarquable, c'est un parcours historiographique dense et passionnant sur l'histoire et l'archéologie du Proche-Orient. En six parties, il dégage les lignes de force du travail d'exhumation et de lecture des traces du passé, en combinant les enjeux thématiques, méthodologiques et les évolutions de la discipline sur une durée de deux siècles environ.

Paru initialement en italien, en 20I3, ce livre est une somme, comme en témoigne la bibliographie finale qui ne compte pas moins de 78 pages. Les sujets abordés sont innombrables afin de décoder les Babylone que l'imagination et le travail critique des historiens ont fait naître ou ont restituées : l'empreinte du témoignage biblique (Babylone, Babel...) dans la reconstruction d'un passé marqué par un imaginaire foncièrement négatif; le poids des lectures colonialistes ou, plus récemment, globalisées, dans la manière de mettre en scène l'Orient dans ses rapports avec l'Occident; le recours problématique à des notions grecques pour appréhender la Mésopotamie, comme l'oikos ou la polis; l'impact de plusieurs formes de déterminismes, géographique, environnemental, économique ou idéologique... Babylone, par son importance, son rayonnement, est le carrefour de nombreux questionnements sur ce qu'est une ville et un processus d'urbanisation, ce qu'est un empire et ses mécanismes de gestion, ce qu'est une révolution et ce que sont les cycles de l'histoire, etc.

Liverani se meut avec la même aisance, la même érudition, avec un regard toujours aiguisé et lucide, de l'Anatolie aux frontières de l'Indus, en passant par Babylone, point focal de l'enquête, dont l'incroyable fortune est responsable de cristallisations historiographiques ici passées au crible fin. Elles engagent essentiellement une science " occidentale» : allemande, britannique, française, américaine, mais aussi italienne, russe, etc. L'analyse est structurée autour de six moments, six étapes du cheminement de la science. De la découverte de Babylone à la post-modernité, dans chaque chapitre, Liverani met en avant les figures marquantes, les idées et les débats, les méthodes nouvelles, les propositions et les procédures, les paradigmes et contreparadigmes. On voit comment l'éventail des méthodes et des concepts s'ouvre, se diversifie par le biais d'une plus grande osmose entre les sciences humaines et sociales, voire au-delà. Avec équilibre et clairvoyance, Liverani montre les avantages et les limites de méthodologies récentes, comme la modélisation spatiale ou la 3D. Il souligne la complexité grandissante de l'herméneutique historienne, mais aussi les écueils inhérents à l'illusion du continuisme. Babylone est-elle une ville ou une non-ville? Babylone est-elle structurée autour de households? Que faut-il entendre 
par « palais » ou par “ temple»? Comment Babylone interagit-elle avec les périphéries? Sur chacun de ces thèmes, Liverani trace le chemin de la recherche, restitue et analyse les apports des courants, des écoles, des personnes.

On suit ainsi les traces de Frankfort, Weber, Childe, Andrae, Jacobsen, Polanyi, Adams, Margueron, Aurenche, McCormick Adams, etc. Une passionnante galerie de portraits, mais aussi et surtout de manières de faire de l'histoire et de l'archéologie, de lire les traces du passé, dans un va-et-vient tantôt fécond, tantôt risqué avec le présent. À partir du cas de “ Babylone », on traverse deux siècles d'histoire intellectuelle dans laquelle le passé est sans cesse réactualisé, dans un mouvement qui pourrait être lu comme un progrès des connaissances, mais qui suggère à Mario Liverani d'autres considérations dans son trop bref “Épilogue ». Il y met en avant la notion de “parabole» comme clé de lecture de tout l'arc parcouru (p. 384-387), en assumant ce qu'une telle lecture peut avoir d'ego-centré. On termine le livre avec la sensation d'une conclusion escamotée, mais renouer, au terme d'un parcours si foisonnant, tous les fils de l'enquête eût été impossible et sans doute inutile.

Ce livre est destiné à intéresser et enrichir un public bien plus large que celui des spécialistes du Proche-Orient ancien. C'est un modèle d'archéologie des savoirs, servi par une fouille minutieuse, attentive aux courants de pensée, aux effets de mode scientifique, aux avancées comme aux impasses. Les apports de l'histoire, de l'archéologie, de l'anthropologie, de la sociologie, des digital humanities même sont combinés et articulés, donnant à voir un paysage intellectuel savamment mis en ordre, mais suffisamment complexe pour éviter tout réductionnisme. Sur le plan épistémologique, les réflexions importantes de Liverani touchent aux conditions de production, de circulation et de reformu- lation des savoirs, autant de sujets que les lecteurs d'Anabases apprécieront.

On peut regretter que la qualité de l'illustration ne soit pas toujours satisfaisante et que la cartographie trouve si peu de place dans un volume qui, depuis Babylone, se déploie sur de très amples territoires. En revanche, on sort de cette longue et exigeante lecture, enrichi et admiratif, convaincu de l'extrême intérêt de ce type d'analyses. Elles requièrent, il faut l'avouer, une expérience et une acuité hors du commun. Imaginer Babylone et se pencher sur les façons dont on l'a pensée, c'est en définitive réfléchir sur les manières d'écrire l'histoire.

\section{Corinne Bonnet \\ Université Toulouse-Jean Jaurès cbonnet@univ-tlse2.fr}

\author{
Françoise-Hélène Massa-Pairault, \\ Claude Pouzadoux (dir.), \\ Géants et Gigantomachie \\ entre Orient et Occident, \\ Naples, Collection du Centre Jean Bérard \\ $\mathrm{n}^{\circ}$ 47, 20I7, $278 \mathrm{p}$., \\ 48 euros / ISBN 978-2-9I8887-74-4.
}

Cet ouvrage constitue la publication collective des actes du colloque organisé par le Centre Jean Bérard (CNRs/EFr) à Naples les I4 et I5 novembre 20I3, sous la direction de Françoise-Hélène Massa-Pairault et de Claude Pouzadoux. Il s'agit de dix-huit articles répartis en quatre axes thématiques: “Les gigantomachies aux époques archaïques et classiques », "Les Géants en Apulie », “ L'époque hellénistique » et « Avatars », qui présentent les diverses interprétations sur le thème iconographique de la Gigantomachie en Grèce, en Italie et en Asie Mineure de l'époque archaïque à l'époque romaine et jusqu'à nos jours. Dans l'introduction, Françoise-Hélène MassaPairault présente l'évolution des figures 
des Géants dans le temps et dans l'espace - Grèce, Italie, Asie Mineure - tout en soulignant le message allégorique inhérent à la Gigantomachie en tant que contestation de l'ordre établi, ainsi que le symbolisme politique dont les Géants ont été porteurs en Grèce et principalement à Athènes, comme le démontrent la fête des Panathénées, le théâtre et les œuvres d'art public.

Dans la première partie, Mario Torelli se concentre sur la perception de la Gigantomachie en Étrurie à travers l'étude des appliques en bronze de chars archaïques. Il attribue l'apparition de ce thème figuratif en Étrurie à la diffusion de la poésie grecque de la seconde moitié du vis ${ }^{\mathrm{e}}$ et du début du $\mathrm{vI}^{\mathrm{e}}$ siècle av. J.-C. dans la région. Dans leur intervention, Mauro Menichetti et Luca Cerchiai s'appliquent à fournir un cadre de référence sur le rapport entre Gigantomachie et rapports agonistiques dans le monde étrusque, en complément de l'intervention de M. Torelli. Ils soulignent l'importante contribution de la céramique $\mathrm{du} \mathrm{VI}^{\mathrm{e}}$ siècle à l'évolution sémantique $\mathrm{du}$ thème pictural vers une signification davantage agonistique. Valentini Nizzo s'intéresse, quant à lui, à une série de représentations vasculaires à figures noires et rouges inspirées de la gigantomachie et découvertes à Spina par P.E. Arias dans les années ig5o. Enfin, Federica Giacobello se consacre à l'étude du cratère 8I52I du Musée Archéologique National (MAN) de Naples représentant la Gigantomachie, daté de la fin du $v^{\text {e }}$ siècle av. J.-C. et attribué au Peintre de Talos.

Dans la seconde partie, Frank Hildebrandt présente deux vases apuliens fragmentaires du Museum für Kunst und Gewerbe à Hambourg décorés de scènes de la Gigantomachie, notamment un cratère à volutes fragmentaire attribué au Peintre de Darius et daté de 340 av. J.-C. L'article suivant de Luca Giuliani présente une étude axée sur l'évolution du motif iconographique de la Gigantomachie en Apulie et sur les innovations apportées par les artistes locaux. Ony soulignera la division de l'espace vasculaire en deux parties symbolisant la terre et le ciel et la mise en valeur des combats individuels entre un dieu et un géant. Eliana Mugione revient sur le symbolisme de la Gigantomachie à Athènes, constituant une allégorie des Guerres Médiques, de la lutte de la civilisation contre la barbarie. Elle évoque l'introduction de la figure de Dionysos dans les représentations de la scène dès l'époque archaïque, soulignant le rôle important que le dieu a progressivement assumé, en tant que figure de l'altérité. Claude Pouzadoux étudie le passage au $\mathrm{Iv}^{\mathrm{e}}$ siècle de ce thème figuratif de la Grèce aux cités-états de l'Italie du Sud qui ont adopté le symbolisme de l'altérité, afin d'exprimer leurs rapports avec les peuples autochtones de leur région.

La partie 3, “L'époque hellénistique », commence avec Évelyne Prioux qui analyse le rôle de la Gigantomachie dans la poésie hellénistique. Son texte met l'accent sur deux questions : les poètes hellénistiques ont-ils en effet composé des œuvres inspirées de la Gigantomachie? Si tel était le cas, sous quelle lumière ont-ils présenté les Géants? Enfin, l'auteur aborde les réflexions esthétiques dont les Géants ont fait l'objet par des poètes de diverses époques, tels Aristophane, Théocrite et Ovide. L'article de Françoise-Hélène Massa-Pairault se propose d'examiner le camée d'Athénion (MAN Naples 25848), alors que dans le même contexte, Filippo Coarelli oriente le débat sur une datation plus basse de l'Autel de Pergame. François Queyrel, pour sa part, présente la transposition de la Gigantomachie dans le contexte des guerres entre les Grecs et les Galates. Les figures des Géants vaincus symbolisent désormais les défaites des Galates qui ont ainsi remplacé les Perses dans l'allégorie politique que le thème a assumée depuis l'époque archaïque. L'article de Pascale Linant de Bellefonds s'intéresse à la figure du géant ailé et anguipède, hybride de nature céleste et chtonienne, qui n'a pas 
survécu très longtemps dans la sphère artistique en raison de la transformation du thème de la Gigantomachie à l'époque romaine en motif générique symbolisant le combat entre l'ordre établi et le chaos et où les Géants se sont transformés en un groupe menaçant anonyme. Enfin, Pierre Leriche présente au lecteur un emblêma mis au jour en 2009 dans l'Ancienne Termez en Bactriane - Ouzbékistan actuel -, qui est décoré de la Gigantomachie. Il procède à la description et à l'analyse de la scène afin d'identifier les personnages représentés par le biais aussi bien des postures et des habits des combattants que de l'étude parallèle d'abord de récits antiques (L'Odyssée d'Homère, la Théogonie d'Hésiode), puis de monuments célèbres (Autel de Pergame).

Dans la conclusion, “Avatars », on soulignera la remarquable contribution de Claude Frontisi qui explore l'émergence de la Gigantomachie à partir des cosmogonies antiques fondées sur le conflit entre les forces primaires - Bien/Mal, Ordre/Chaos. Il évoque la transposition du motif antique dans l'art de la Renaissance à travers la revivification de l'intérêt émanant des érudits de l'époque - auteurs, philosophes et artistes - envers les textes et les vestiges antiques qui se focalisent sur les célèbres Géants, autres que ceux ayant participé au combat contre les Olympiens, comme Polyphème et Goliath, et surtout Orion.

Les actes du colloque sont accompagnés d'un court catalogue de l'exposition "Géants et gigantomachies entre Orient et Occident " présentée au Musée National Archéologique de Naples entre novembre 2013 et mars 20I4 sous l'égide de nombre d'institutions italiennes et françaises. L'ouvrage comprend les quinze panneaux et notices didactiques présentés lors de l'exposition, accompagnés d'un riche matériel iconographique (œuvres d'art, inscriptions, cartes) qui aidaient le visiteur à suivre l'évolution du mythe des Géants et son symbolisme dans le temps $\left(\mathrm{du} \mathrm{vI}^{\mathrm{e}}\right.$ siècle av. J.-C. au $v^{e}$ siècle ap. J.-C.) et l'espace
(Grèce, Italie, Asie Mineure), à travers des exemples caractéristiques de la littérature et des arts antiques associés à divers aspects de la vie (symposion, politique, culte, sépulture).

Cette publication du Centre Bérard offre un remarquable panel d'un motif iconographique qui a marqué auteurs et artistes de l'Antiquité à nos jours. D'une très haute qualité visuelle, l'ouvrage propose des études approfondies accessibles à un large public et touche des domaines aussi variés que la littérature, l'art, la cosmographie, la religion. Une lecture passionnante qui séduira aussi bien les amateurs d'art que les spécialistes du sujet de la Gigantomachie.

$$
\begin{array}{r}
\text { Carine GiovÉNAL } \\
\text { Université François-Rabelais de Tours } \\
\text { c.giovenal@hotmail.com }
\end{array}
$$

Scott McGill, Joseph Pucci (éd.), Classics renewed. Reception and Innovation in the Latin Poetry ofLate Antiquity,

Heidelberg, Universitätsverlag Winter, Bibliothek der klassischen

Altertumswissenschaften, hrsg von J. Paul Schwindt, Neue Folge 2.Reihe, Band I52, 20I6, 43o p., 48 euros /ISBN 9783825364489 .

Cet ouvrage extrêmement stimulant regroupe une sélection de travaux présentés originellement lors d'un colloque en deux parties qui s'est tenu à Rice University et à Brown University en 20II : la sélection s'est faite autour de la question de la réception des textes classiques dans l'Antiquité tardive; les deux derniers articles [voir le sommaire infra] excèdent ces limites, mais ils forment un prolongement intéressant du sujet puisqu'il y est question de l'époque carolingienne et du rapport qu'elle entretient elle-même avec les œuvres de l'Antiquité et classique et tardive. 
I5 contributions au total, sur des auteurs variés (seul Claudien apparaît dans trois chapitres, mais les textes ou les passages considérés, ainsi que les questionnements, diffèrent; les types de textes étudiés sont également variés: ainsi, la poésie épigraphique est mise à l'honneur dans la contribution de D. Trout), qui permettent au lecteur moins familier de l'Antiquité tardive d'y faire un « bain » de poésie très instructif et enrichissant, surtout si ce lecteur ou cette lectrice s'intéresse aux problématiques de la réception et de l'intertextualité.

Conformément à ce qu'annoncent son titre et son sous-titre, un des maîtresmots de l'ouvrage est la nouveauté propre à la poésie latine tardive : la présence des auteurs classiques dans les œuvres est importante, mais celles-ci apparaissent comme dominées par la volonté de créer une esthétique nouvelle, d'exprimer de nouvelles façons de penser, de renouveler profondément des genres poétiques donnés (l'épopée de Juvencus étudiée par S. Mc Gill, par exemple, épopée véridique, qui adopte néanmoins Virgile comme son modèle-code et fait, en tout cas, sien le langage virgilien, se distingue absolument de tout précédent épique parce qu'elle est la première à transformer les Évangiles en poèmes narratifs en hexamètres; la bucolique dans le Carmen de mortibus boum d'Endelechius - au cœur de l'étude de P.Schierl-s'écrit dans le mètre lyrique horatien de manière à être constituée de trente-trois strophes (“christiques »). Le contexte poussait clairement à un tel renouvellement: le christianisme constituait un nouveau cadre culturel, que les auteurs non chrétiens ne pouvaient pas ignorer. Pour autant, l'attitude vis-à-vis des modèles classiques apparaît comme n'étant pas seulement faite d'opposition ou de subversion (un exemple de Kontrastimitation est néanmoins brillamment présenté par E.J. Hutchinson à propos du Carmen Paschale de Sedulius). La variété des cas d'étude et, peut-être aussi, des références intertextuelles propres à chaque contribu- teur, fait qu'on obtient une vue complexe, en aucun cas schématique : comme l'oppositio in imitando, le fameux « Jeweled style » ne caractérise pas au même degré toutes les œuvres de la période considérée ; ainsi, selon l'analyse de S. Schottenius Cullhed, un trait majeur du rapport que Proba, dans son centon virgilien, entretient à la fois avec Virgile et la Bible est la recherche d'unification; le modèle virgilien est alors vu comme intégré à une construction qui respecte la nécessité de cohérence du centon selon Ausone.

Un enjeu majeur du livre consiste à renforcer l'idée d'une spécificité de la poésie latine tardive, en particulier dans la relation qu'elle entretient avec ses modèles et dans les pratiques et procédés intertextuels liés: les notions utilisées par les différents contributeurs ne sont pas toutes nouvelles (on retrouve par exemple souvent les noms de S. Hinds et de R.F. Thomas, dont les travaux ont été et demeurent si importants pour l'analyse et l'appréciation de l'intertextualité dans la poésie augustéenne). Mais en écho à la remarque de $\mathrm{M}$. Formisano rappelée en introduction (р. เ6), selon laquelle cette poésie latine tardive si nouvelle exige parfois de nouveaux outils d'analyse, des notions - relativement - moins courantes sont aussi utilisées dans le volume, comme l'idée d'allusions référentielles vs non-référentielles, le concept d'imitation itérative (central dans l'article particulièrement stimulant de B. Mulligan, qui reconsidère d'ailleurs utilement le lien étroit entre traduction et imitation).

Si de rares critiques doivent être faites au volume, l'une tient peut-être au fait qu'on n'est pas toujours absolument convaincu par l'idée d'une spécificité des démarches intertextuelles ou allusives étudiées. À plusieurs reprises, toutefois, cette objection est anticipée et des arguments supplémentaires sont avancés, qui consistent notamment à tenir compte du contexte et d'un cadre de référence 
qui rendent nécessairement les procédés en question différents. Par ailleurs, les critères de repérage des allusions auraient parfois gagné à être explicités de manière à rendre celles-ci plus sûres et, par là encore, à faire apparaître leur différence par rapport aux allusions que pratiquaient les poètes classiques. Ce manque est cependant tout relatif parce qu'une des très grandes qualités de l'ouvrage consiste dans ses apports méthodologiques: plusieurs auteurs du volume balisent, discutent, nuancent les lectures qu'ils font des allusions et intertextes d'une manière qui est à la fois honnête, lucide, claire et en définitive exemplaire et utile.

Séverine Clément-Tarantino Université de Lille sHS ; HALMA, UMR 8I64 severine.tarantino@univ-lille3.fr

Maxwell T. Paule, Canidia, Rome's First Witch, Londres - New York, Bloomsbury Academic, 20I7, 2I8 p., II/ dollars / ISBN 978I350oo3880.

L'évocation des sorcières ne fait pas d'emblée penser à l'Antiquité. Pourtant le monde gréco-romain a conservé le souvenir de figures incontournables et exceptionnelles telles Médée ou Erichto. D'autres personnages sont réputés secondaires, comme Canidia, même si c'est un personnage récurrent des poèmes d'Horace, une “dark anti-muse " selon l'auteur (p. I). La complexité de Canidia apparaît dans les Épodes et les Satires. Figure tantôt inquiétante tantôt risible, elle tue, empoisonne, pratique des rituels magiques... Cependant l'historiographie a longtemps négligé une étude exhaustive $\mathrm{du}$ personnage, c'est pourquoi l'auteur propose ici une analyse systématique en cinq chapitres des occurrences de Canidia dans l'œuvre d'Horace.
Le premier chapitre (Canidia, or what is a witch?) fait d'abord office d'introduction et permet de justifier les choix de M. T. Paule. L'identification à Gratidia, une parfumeuse originaire de Naples, est ainsi d'emblée réfutée. Canidia serait donc bien un personnage fictif. Ensuite, contrairement aux idées reçues, les sorcières romaines ne formaient pas une catégorie uniforme et l'auteur veut démontrer que chaque apparition de Canidia dans les poèmes d'Horace offre une facette différente de la précédente. Cette « demonic figure » (p. 2) sait s'adapter à toutes les situations. M. T. Paule compare alors les sorcières antiques à des figures démoniaques. Ce chapitre est d'ailleurs plus largement l'occasion de faire le point sur les sorcières dans la littérature latine. Le terme générique de “witch» est trop vague par rapport à la terminologie latine qui décrit plus précisément des détentrices de pouvoirs spécifiques (anus, lamia, maga, saga, uenefica...). Le vocabulaire latin est étudié afin de dégager la complexité de la figure de la sorcière.

Dans le deuxième chapitre (Satire I.8: Canidia in the gardens of Maecenas), Canidia est dépeinte comme une intruse et une magicienne peu crédible, avant d'être chassée des Jardins de Mécène par une statue de Priape. Plusieurs niveaux de lecture sont possibles. D'abord en utilisant les horti comme un cimetière pour ses rites magiques, elle remettait en cause l'espace même des jardins alors en quête de pureté. En effet le terrain acquis récemment par Mécène souffrait de son passé de cimetière. Puis l'expulsion de l'intruse doit être vue comme une "metanarrative of Horace's own poetic struggles with the composition of the Satires » (p. 20). C'est effectivement une métaphore du travail poétique avec les défis à relever par le poète pour créer une nouvelle forme de satire.

Le chapitre suivant (Hag and snatcher: Canidia as child-killing demon in Epode 5) étudie sa facette la plus épouvantable, celle rappelant les anciennes entités démo- 
niaques tueuses d'enfants (Lilith dans l'Orient ancien, Lamia dans le monde grec et la strix à Rome). Canidia prévoit l'enlèvement et le meurtre d'un enfant. L'auteur établit alors des liens entre la Cinquième épode et la Quatrième églogue de Virgile mettant en scène la naissance à venir d'un enfant et l'arrivée de l'âge d'or. L'auteur analyse de manière claire et précise les comparaisons (p. 79-87), car le poème d'Horace pourrait être perçu comme une réponse aux attentes d'une paix à venir prédite par Virgile dans son églogue.

Le dernier poème des Épodes laisse encore apparaître une autre Canidia, une sorte de séductrice tueuse et torturant Horace. La figure de l'empusa est donc analysée dans le quatrième chapitre (Routing the empusa: the iambic Canidia of Epode I7). La sorcière serait en fait selon l'auteur la personnification féminine des Épodes d'Horace. Enfin le dernier chapitre s'intéresse aux autres mentions mineures de Canidia mettant en lumière ses capacités d'empoisonneuse: la préparation des poisons dans la Troisième épode, l'utilisation de l'Albuci uenenum dans la Satire, 2, I et la réputation d'un souffle mortel dans la Satire, 2, 8.

Pour conclure, le simple terme de “witch" semble trop réducteur pour décrire la complexité du personnage. C'est pourquoi Canidia occupe une place à part parmi les grandes figures de la sorcellerie dans l'Antiquité et l'ouvrage a bien montré le caractère pluriel et mouvant du personnage. Même si le public visé est d'emblée celui des spécialistes d'Horace, l'ouvrage se veut très accessible, y compris pour des étudiants. L'accessibilité est notamment facilitée par des propos clairs et résumés à chaque fin de chapitre. Pour faciliter l'immersion dans le texte poétique, les vers latins sont toujours assortis d'une traduction anglaise. L'ensemble est aussi accompagné de notes regroupées à la fin de l'ouvrage (p. I5I-ı98), ce qui rend hélas leur consultation malaisée. Le lecteur trouvera également une bibliographie en grande majorité en langue anglaise (p. I99-2I3) et un index général (p. 2I5-2I8).

Cyrielle LANDREA

Université Bretagne Sud cyrielle.landrea@univ-ubs.fr

Jessica Priestrey, Vasiliki Zali (éd.), Brill's Companion to the Reception of Herodotus in Antiquity and Beyond, Leiden, Brill, 2or6, xvi+438 p., I53 euros / IsBN 978-90-04-27229-3.

Malgré son titre, le volume édité par Priestley et Zali n'est pas à proprement parler un Companion, si l'on entend du moins par là une forme d'aperçu général d'un sujet donné tendant, autant que possible, à l'exhaustivité. Non, il s'agit bien ici d'une collection d'articles, multipliant les éclairages particuliers autour du thème de la réception d'Hérodote, mais sans intention de couvrir l'intégralité du champ scientifique ni même d'offrir réellement une introduction ou un guide sur le sujet (comme ce semble pourtant être le but des éditeurs, p. I3). Ceci se reflète assez clairement dans la délimitation chronologique assez floue contenue dans le titre : « in Antiquity and Beyond ». En effet, si le livre rassemble à proportion quasiment égale des contributions sur la réception d'Hérodote dans l'Antiquité et aux époques ultérieures, c'est pour ces dernières que le déséquilibre est le plus marqué et que la différence entre une somme d'approches particulières et un Companion se fait le plus sentir. Ainsi, l'Antiquité grecque tardive (le monde latin reçoit quelque attention) et surtout le monde byzantin sont totalement absents (les regrets exprimés p. I4 sur l'état des recherches sur la réception d'Hérodote ne justifient pas totalement ces absences). Ceci étant dit, les articles rassemblés dans ce volume sont tout à fait 
remarquables et proposent une approche très large de la réception d'Hérodote, dans la ligne de nombreux travaux récents (détaillés p.3). Chacune de ces approches montre qu'Hérodote, comme Thucydide, occupe une place toujours plus importante dans le discours occidental sur l'histoire, précisément parce que son œuvre domine la tradition, dans une proportion que les débats à son propos ne font que renforcer, dès l'Antiquité.

Dans un article quelque peu spéculatif, M. Wecowski (I. Herodotus in Thucydides: A Hypothesis) émet l'hypothèse que la différence fondamentale entre les ouvres d'Hérodote et de Thucydide (et entre celles-ci et les Hellenica de Xénophon dans un second temps) est corrélée à l'évolution du contexte culturel dans lequel elles ont été produites et, partant, dépend de l'attente supposée de leurs audiences respectives. Les idées très séduisantes exprimées dans ces pages auraient gagné à être étayées par une analyse plus détaillée. V. Zali pour sa part aborde le même corpus sous un autre angle (2. Herodotus and His Successors: The Rhetoric of the Persian Wars in Thucydides and Xenophon): dans quelle mesure les allusions aux Guerres Médiques chez Thucydide et Xénophon révèlent leur positionnement vis-à-vis des Histoires d'Hérodote? Abordant spécialement la question del'unité des Grecs face à l'ennemi, Zali montre comment le paradigme hérodotéen nourrit la manière dont ses successeurs déploient les références au passé pour explorer les possibilités de l'avenir. Dans une étude solide (3. Duris of Samos and a Herodotean Model for Writing History), Ch. Baron montre pour sa part que l'influence d'Hérodote sur Douris de Samos aide à la fois à comprendre le projet historiographique de celui-ci, malgré l'état fragmentaire dans lequel nous est parvenue son œuvre, mais aussi, en retour, ce que cela signifiait d'écrire une histoire à la manière d'Hérodote à l'époque hellénistique. E. Almagor (4. "This is What Herodotus
Relates": The Presence ofHerodotus' Histories in Josephus' Writings) montre que Flavius Josèphe, à son tour, inscrit son œuvre dans la continuité de celle d'Hérodote. Sans ignorer les critiques adressées à son illustre prédécesseur au fil de la tradition historiographique, et non sans le corriger ou le compléter lui-même, Flavius Josèphe se montre ainsi profondément influencé par le paradigme hérodotéen, qu'il s'agisse de langue, de style narratif, ou de conception de l'histoire. L'attitude de Plutarque par rapport à Hérodote est, elle, plus ambigüe qu'il n'y paraît. Comme le montre Marincola (5. History without Malice: Plutarch Rewrites the Battle of Plataea), malgré les critiques qu'il lui adresse dans son de Herodoti malignitate, Plutarque suit globalement le récit hérodotéen quand il en vient à relater dans les Vies les événements des Guerres médiques, mais en ajoutant à ce récit une dimension morale (propre au genre biographique, mais également présente chez un historien comme Polybe par ex.) et dans le but évident de le surpasser.

Toujours dans la même section, l'article de B. Earley (6. Herodotus in Renaissance France) examine les différentes lectures d'Hérodote dans la France du xvi ${ }^{\mathrm{e}}$ s., alors que l'Europe prémoderne voit émerger différentes philosophies de l'histoire. La réhabilitation d'Hérodote comme auteur véridique par Estienne (Apologia pro Herodoto, I566) ne doit pas occulter d'autres attitudes, tantôt intéressées à la portée morale du texte et non à sa factualité, tantôt franchement critiques, tantôt plus respectueuses de la place d'Hérodote dans le développement de l'historiographie. N. Morley à son tour (7. The Anti-Thucydides: Herodotus and the Development of Modern Historiography) montre que l'opposition entre Hérodote et Thucydide comme représentants de deux approches opposées de l'historiographie est une construction relativement récente dans la réception de ces deux auteurs, consécutive au développement d'une 
approche scientifique de l'histoire au $\mathrm{xIx}^{\mathrm{e}} \mathrm{s}$. qui voit en Thucydide son modèle. Malgré un retour en grâce d'Hérodote, à partir du début du $\mathrm{xx}^{\mathrm{e}} \mathrm{s}$., comme père fondateur de l'historiographie, et la mise en cause de l'objectivité longtemps attribuée à Thucydide, ce dernier demeure dans la tradition occidentale un modèle de rigueur, à l'opposé de son prédécesseur.

L'article d'O. Tribulato (8. Herodotus' Reception in Ancient Greek Lexicography and Grammar: From the Hellenistic to the Imperial Age) ouvre une nouvelle section et montre de manière très fine comment les références à Hérodote dans les ouvrages antiques de lexicographie nous informent sur la réception de cet auteur dont la langue devient un modèle en raison de l'œuvre où elle se déploie et de la portée panhellénique de son message et de son thème.

F. Racine (9. Herodotus' Reputation in Latin Literature from Cicero to the $\mathrm{I}^{\text {th }} \mathrm{Cen}$ tury) s'intéresse à la réputation d'Hérodote chez les auteurs latins avant le $\mathrm{xII}^{\mathrm{e}} \mathrm{s}$. : si de nombreuses anecdotes remontant, en définitive, au texte d'Hérodote ainsi que le nom de l'historien lui-même sont connus, c'est le plus souvent de manière indirecte. Cela n'empêche que, durant douze siècles, Hérodote apparaît à la fois comme un modèle stylistique et comme une source d'information digne d'être nommée. A. Foley donne pour sa part la suite de cette histoire (Io. Valla's Herodotean Labours: Towards a New View of Herodotus in the Italian Renaissance) et s'intéresse au contexte dans lequel Lorenzo Valla a donné sa traduction latine d'Hérodote, conçue d'abord comme l'appropriation d'une œuvre du patrimoine grecque dans la romanitas du xve siècle. D. Looney (II. Herodotus and Narrative Art in Renaissance Ferrara: The Translation of Matteo Maria Boiardo) montre pour sa part que la première traduction d'Hérodote en italien par le poète Matteo Maria Boiardo remplit, elle aussi, un rôle avant tout esthétique, comme modèle narratif, et non historiographique.
L'analyse d'A. Schwab (I2. The 'Rediscovery' of Egypt: Herodotus and His Account of Egypt in the Voyage dans la Basse et la Haute-Égypte (I802) by Vivant Denon) révèle quant à elle comment Vivant Denon, tout en se positionnant par rapport à Hérodote en imitateur et compétiteur, parvient à s'effacer lui-même de sa narration.

B.E. Stevens (13. Not beyond Herodotus? Psammetichus' Experiment and Modern Thought about Language) s'intéresse, dans un tout autre genre, à la réception dans le discours linguistique prémoderne et moderne de l'، expérience interdite » menée sur ordre de Psammétique pour explorer les origines du langage (isoler deux enfants en bas âge de tout langage humain pour identifier la langue originelle) et qui jusqu'à aujourd'hui suscite la fascination.

Enfin, dans une dernière section, V. Gray (I4. Herodotus (and Ctesias) Re-enacted: Leadership in Xenophon's Cyropaedia) montre comment Xénophon a réinterprété la figure de Cyrus dans un cadre socratique, mais pas uniquement à partir d'Hérodote, dont l'influence ici ne doit pas être exagérée. Pausanias et sa relation aux Histoires sont au cœur de l'article de l'article de G. Hawes (I5. Pausanias and the Footsteps of Herodotus); il apparait que le Périégète s'est approprié le modèle hérodotéen, tant dans le style que dans la narration, pour organiser sa matière et affirmer son autorité particulière comme voyageur et narrateur.

L'analyse de deux relectures des Histoires d'Hérodote dans la littérature contemporaine ferment le volume. L'essai autobiographique du reporter polonais Ryszard Kapuściński (1932-2007) est entrelacée, jusque dans son titre, de référence aux Histoires et l'auteur luimême se veut un double de l'historien, $\mathrm{vu}$ avant tout comme un voyageur. Ainsi, comme le montre K. Kosmala (I6. Ryszard Kapuścinski's Travels with Herodotus: Reportagefrom theSelf), c'est une vision bien particulière d'Hérodote que Kapuściński propose, façonnée surtout par l'image 
qu'il veut donner de lui-même, fascinante création littéraire donc, à mi-chemin entre deux réalités évanescentes. Heather Neilson pour sa part (17. Herodotus in Fiction: Gore Vidal's Creation) s'intéresse au roman épique Creation de Gore Vidal qui revisite à la marge nombre de récits connus par Hérodote. À défaut de problématique clairement identifiée, ce dernier essai permet surtout de se familiariser avec l'œuvre de Vidal.

Les éditeurs n’ont donc pas adopté une approche strictement chronologique, mais ont réparti les textes en trois sections: “Father of History» (articles I à 7), “Language, Translation and Scholarship " (8 à I3), enfin “ New Narratives and Genres » (I4 à I7). La distribution des articles dans ces catégories semble cependant quelque peu artificielle. Le lecteur est bien entendu libre de se faire son propre parcours dans le livre (ainsi en groupant la lecture des articles 6 , го et II ou I2 et I6), mais cette répartition induit une vision de la réception d'Hérodote qui me semble incorrecte. Si l'essai de Kapuściński ou le roman de Vidal peuvent, effectivement, ressortir à la catégorie des “ New Narratives and Genres », il me semble que la Cyropédie et la Périégèse s'inscrivent plus nettement dans la continuité des Histoires, et cette forme de réception révèle, précisément, le caractère dynamique de l'historiographie comme genre (ou ensemble de genres) dans l'Antiquité, comme le montre aussi l'article sur Plutarque, intégré pour sa part dans la première section. Moyennant ces réserves, on ne peut que saluer la parution de ce volume de grande qualité qui ouvre de nombreuses perspectives de recherche.

Olivier GENGLER

Université de Vienne ogengler@yahoo.fr
Salvatore QuAsimodo,

LaLyre grecque,

Traduit de l'italien et postfacé

par Patrick Reumaux,

Éditions Vagabonde, 2018, 216 p.,

2I,50 euros / ISBN 978-2-9I9067-25-I.

En I940, lorsque les éditions Corrente publient l'œuvre Lirici greci de S. Quasimodo (I9oI-I968), poète et traducteur, une des voix majeures de la poésie italienne $\mathrm{du} \mathrm{xx}^{\mathrm{e}}$ siècle, les réactions des philologues, des fidèles des traductions pédantes ne tardent pas, pas plus que celles des détracteurs à l'égard de celui qui avait appris le grec ancien tardivement et presque en autodidacte. En ouvrant le volume La Lyre grecque, on trouve la préface signée par Quasimodo et des éclaircissements : ses traductions se sont libérées des chaînes métriques originelles pour trouver "la cadence interne de la parole érigée en vers ». Et il ajoute: “ Je parle de la véritable quantité contenue dans chaque parole (dans la tournure de la voix qui la prononce) ». Patrick Reumaux, poète, romancier et traducteur français, relève le défi et fait connaître la sélection des lyriques grecs qu'avait opérée Quasimodo, en les traduisant de l'italien au français pour la première fois, avec des résultants qui ne sont pas moins remarquables. La galerie des élus comprend Sappho et Alcée de Lesbos, Érinna de Tilos, Anacréon de Téos, Alcman de Sardes, Stésichore d'Himère, Ibycos de Rhégion, pour citer certains des auteurs les plus connus, sans oublier quelques anonymes. Pour certains auteurs une seule page suffit, voire une seule composition, comme pour Stésichore d'Himère, tandis que de Sappho sont présents une vingtaine de poèmes. Si dans le passage du grec à l'italien Quasimodo avait fait preuve de tant d'audace et de liberté qu'on peut parler de nouvelles créations, autonomes, bref de poèmes de Quasimodo, les traductions de Reumaux sont davantage proches du référent italien. Mais il s'agit de proximité 
dans la distance, conformément à une vraie théorisation de la traduction que Reumaux illustre dans la postface signée de sa main. Les exemples jalonnent le volume. Sappho ouvre le répertoire avec une composition, "À Aphrodite ", où, dès la première strophe, on peut observer le décalage, si subtil, des traductions : " tu che non hai morte / figlia di Zeus, tu che intrecci inganni, / o dominatrice, ti supplico, / non forzare l'anima mia / con affanni né con dolore ». De ces tourments et douleurs Reumaux, de son côté, demande à être épargné “ je t'en supplie, / épargne à mon âme / tourments et douleurs ». Le texte grec présente le verbe $\delta \alpha \mu v \alpha ́ \omega=\delta \alpha \mu \alpha ́ \zeta \omega$, signifiant proprement " dompter, soumettre », ce que l'italien a rendu par "forzare ", tandis que le français s'en éloigne complètement avec " épargner » qui exprime le sens général de la supplique adressée à la déesse, sans y préciser que les maux d'amour ont l'effet de plier l'âme. Dans un autre poème de Sappho, "Invitation à l'extase ", au "grato bosco» du temple sacré des vierges correspond le "creux du bosquet » en français. Quasimodo choisit un adjectif polysémique, grato, car il signifie à la fois agréable et reconnaissant, tandis que Reumaux recourt à l'image du creux du bosquet, qui renvoie à un endroit reculé où la végétation est dense et luxuriante.

Le travail de Quasimodo d'abord et de Reumaux ensuite est ambitieux, car la matière à traiter se compose de traits rapides, d'images fugaces, celles des poètes lyriques grecs souvent comparés aux peintres impressionnistes. On y retrouve les nuances et les teintes des saisons de la vie. Quasimodo, le premier, s'approprie cette matière, ce “don ", et Reumaux lui vole le butin. C'est en termes de don, vol et embuscade que Reumaux décrit l'opération de traduire. D'Anacréon de Téos, on entend en italien qu'il touche doucement la cithare pour chanter son amour « l'amata cetra tocco con dolcezza e canto amore alla mia tenera fanciulla "; Reumaux, en revanche, par le bais d'une métonymie, ramène la douceur du syntagme adverbial "con dolcezza" au chant d'amour, aux doux accords : “ je tire maintenant de doux accords de ma cithare aimée / pour chanter l'amour à ma tendre mie ». Plus loin, un poème d'Anacréon, “ À un jeune homme mort pour la patrie ", met en lumière le décalage des perceptions et la singularité de la création poétique des deux auteurs. “ Ont mis fin les vagues à ton adolescence» : lapidaire, comme une stèle en mémoire de quelqu'un, un cénotaphe en l'occurrence, cette tournure livre Cléanoride à l'éternité du souvenir. Quasimodo avait accompagné la mort prématurée du jeune Cléanoride d'un geste, " le onde del mare chiusero la tua adolescenza " respectueux, comme si la mer, avec pudeur, scellait une mort dont elle-même était coupable.

“ Faire de l'acte de traduire la répétition d'une différence », c'est aussi en ces termes que Reumaux parle de la traduction dans la postface. D'une différence à l'autre, d'une traduction à l'autre, cependant, un noyau est préservé, ajoute-t-il; il s'agit de la "consistance », de l'objet du vol. Au départ des mots, de leur durée intrinsèque, le traducteur s'approprie les rythmes, les images, car l'opération est synesthésique, et continue de battre le rythme sur son propre chemin. Deux voies parallèles, qui se rapprochent tout en gardant une distance, celle de la traduction. On termine la lecture de ce précieux volume avec l'impression d'avoir suivi les deux auteurs tout au long de leur chemin, pendant qu'ils accomplissaient “le saint labeur de la poésie ».

D’un côté S. Quasimodo face aux poètes lyriques grecs dont il fait revivre les vers, de l'autre P. Reumaux face à S. Quasimodo qu'il traduit en français. Ce travail passe par les mots : quand bien même on tente de les rapprocher d'une langue à l'autre, comme dans un miroir, il demeure autre chose, un autre son, un autre rythme, une musicalité différente. Le texte grec est cependant le grand absent de cette édition. On aurait 
voulu pouvoir le lire dans ces pages, pour mieux saisir la polyphonie des opérations artistiques.

$$
\begin{array}{r}
\text { Maria BIANco } \\
\text { Université Toulouse-Jean Jaurès } \\
\text { maria.bianco@univ-tlse2.fr }
\end{array}
$$

\author{
Brett M. Rogers, Benjamin Eldon \\ Stevens (éd.), \\ Classical Traditions in Modern Fantasy, \\ Oxford - New York : Oxford University \\ Press, 2017, 367 p., \\ 27, 99 livres / sBN 9780igo6ioo67.
}

Après Classical Traditions in Science Fiction, B.M. Rogers et B.E. Stevens éditent un deuxième recueil de $\mathrm{I} 4$ articles sur la “ modern fantasy », sorte d'hydre littéraire de notre temps, ou du moins depuis $L e$ Château d'Otrante d'Horace Walpole, dans lequel se côtoient l'invraisemblance et l'imaginaire, mais qui peut tout à fait être déjà visible chez les auteurs anciens. L'introduction très claire démontre tous les éléments de l'appareil critique sur la littérature fantastique qui est développée en 4 parties distinctes.

La première, "Classical Apparitions in (Pre-)Modern Fantasy ", se compose de 4 essais portant à la fois sur l'épique ou l'épopée en convoquant Virgile, Homère ou Apollonios pour Jesse Weiner dans Classical Epic and the Poetics of Modern Fantasy; le Romantisme allemand et l'inspiration apportée par Lucien de Samosate pour Cecilie Flugt dans Theorizing Fantasy: Enchantment, Parody and the Classical Tradition; le mouvement pré-raphaélite en lien avec les auteurs anglais Lewis ou Tolkien dans The Mirror Crack'd: fractured Classicisms in the Pre-Raphaelites and Victorian Illustration de Genevieve $\mathrm{S}$. Gessert; et le thème horrifique par l'intermédiaire de Lovecraft dans Classical Antiquity and the Timeless Horrors of
H.P. Lovecraft de Robinson P. Krämer. L'ensemble de ces articles exposent des éléments méthodologiques et théoriques avec lesquels le lecteur peut décrypter les romans pré-modernes comme ceux de Tolkien. Il s'agit pour les auteurs d'insister sur ce qu'est la fantasy et ce qu'elle doit à l'Antiquité dans les œuvres des auteurs cités mais aussi sur les processus créatifs des écrivains.

La deuxième partie, “False Medievalism and other Ancient Fantasies ", propose de décortiquer grâce à 3 articles le thème de l'Antiquité cachée dans une fantasy décrite comme "médiévale», par l'intermédiaire des auteurs emblématiques Lewis et Tolkien. Ce dernier est abordé à la fois dans le texte de Benjamin E. Stevens dans Ancient Underworld in J.R.R. Tolkien's The Hobbit alors que deux textes sont consacrés à Lewis, le premier par Jeffrey T. Winkle, C.S. Lewis's The Voyage of the Dawn. Treader and Apuleius's Metamorphoses et le second par Marcus Folch dans A Time for Fantasy: retelling Apuleius in C.S. Lewis's Till We Have Faces. Les thèmes abordés sont ceux de la catabase, des mondes souterrains et les métamorphoses d'Apulée. Sous un vernis de chevalerie visuelle, les auteurs démontrent la persistance de l'Antiquité chez les deux grands auteurs du milieu du $\mathrm{xx}^{\mathrm{e}} \mathrm{s}$.

La troisième partie, quant à elle, est composée de 4 articles, résumée sous l'intitulé "Children and (Other) Ancient Monsters ". Les propositions sont axées autour d'Harry Potter, Á la croisée des mondes et Rebelle de Disney. Cette thématique s'ouvre sur un article général de Sarah Annes Brown, The Classical Pantheon in Children's Fantasy Literature, qui présente les influences des mythes anciens (nordiques et classiques) dans la littérature de jeunesse et l'usage du panthéon, et comment ils sont transformés, réutilisés notamment en Amérique du nord. Dans un univers à la fois antique et fantastique c'est surtout le problème de la transgression des règles par les enfants 
dont il est question dans les articles de Brett M. Rogers Orestes and the Half-Blood Prince: Ghosts of Aeschylus in the Harry Potter Series, d'Antonia Syson dans Filthy Harpies and Fictive Knowledge in Philip Pullman's His Dark Material Trilogy et celui d'Elisabeth A. Manwell dans Girl in Bear's Clothing in Greek Myth and Disney/Pixar's Brave. Cette émancipation passe à la fois par le rite de passage et la confrontation à l'univers de l'adulte comme pour le rite du Brauron ou celui de l'Orestie.

La dernière partie de l'ouvrage, (Post) Modern Fantasies of Antiquity, regroupe trois articles assez différents prenant comme sujet une poésie contemporaine présentée par Sasha-Mae Eccleston dans Fantasies of Mimnernos in Anne Carson's The Brainsex Painting (Plainwater), un roman d'une auteure contemporaine par Jennifer A. Rea dans Aeneas' American New World in Jo Graham's Black Ship et Game of Thrones par Avelet Haimson Lushkov dans Genre, Mimesis, and Virgilian Intertext in George R.R. Martin's A song of Ice and fire, pour exprimer le réemploi de thèmes abordés par Virgile ou le poète grec archaïque Mimnerme. Les thèmes choisis cherchent à démontrer que la réception de l'Antiquité s'exprime dans des lieux divers, en lien avec notre vie quotidienne: politique américaine, question du genre ou du concept de la réalité.

Classical Traditions in Modern Fantasies possède les défauts de ses qualités. La volonté d'aborder un sujet aussi vaste avec un échantillon de thèmes aussi éclectique ne permet pas de rassurer le lecteur néophyte sur la question de la réception. Il est évident qu'un tel ouvrage n'est pas destiné à n'importe qui, tant certains articles demandent un fond de connaissances à la fois sur la littérature et sur l'Antiquité. Cet ouvrage explore des territoires immenses dont les contours sont malheureusement peu lisibles à cause des thèmes retenus. Il a néanmoins le courage de se positionner dans un champ de la recherche encore un peu frileux de la réception de l'Antiquité pour les spécialistes ou non de l'Antiquité.

Matthieu Scapin

Musée Saint-Raymond, Musée des Antiques de Toulouse mathieu.scapin@gmail.com
Maria Teresa Schettino et Céline UrLacher-Becht (dir.), Ipse dixit. L'autorité intellectuelle des Anciens : affirmation, appropriations, détournements,

Besançon, Presses Universitaires de Franche-Comté, Institut des sciences et techniques de l'Antiquité, 20I7, 234 p., I6 euros / ISBN 978-2-84867-577-0.

La question de l'autorité des Anciens et de ses diverses manifestations dans la littérature antique de même que ses réceptions aux époques ultérieures fait l'objet d'un intérêt croissant dans le champ des études anciennes, comme en témoigne la multiplication des publications depuis une dizaine d'années. Sans chercher l'exhaustivité, on notera l'ouvrage de P. Payen et D. Foucault (Les autorités: dynamiques et mutations d'une figure de référence à l'Antiquité, Grenoble : Éd. J. Millon, DL 2007) et celui de M. Gorea et M. Tardieu (Autorité des auteurs antiques: entre anonymat, masques et authenticité, Turnhout, 20I4), de même que les travaux du laboratoire junior de l'ENs Expressions et Représentations de l'Autorité dans les Mondes Anciens (ERAMA) ${ }^{6}$ ou encore le très récent réseau international de chercheurs “Claiming the classical " ${ }^{7}$ qui prend pour objet l'autorité de l'Antiquité classique et

6 http://erama.ens-lyon.fr/spip. php?rubrique1

7 https:/claiming-the-classical.org/ 
ses (més)usages dans la rhétorique politique du xxI ${ }^{\mathrm{e}}$ siècle.

L'ouvrage est issu d'un cycle de conférences établi par Maria Teresa Schettino et Céline Urlacher-Becht qui se sont tenues en 2013 et 20I4 dans divers lieux culturels de l'Alsace afin, comme le souligne cette dernière, d'œuvrer “plus largement encore que dans notre quotidien d'enseignant-chercheur à la transmission de l'héritage antique». L'ouvrage de 234 pages se compose de deux parties : I) Nova sed nove, le détournement de l'autorité des Anciens dans l'Antiquité tardive et II) L'auctoritas des historiens à l'époque humaniste, précédées d'un Avant-propos et d'une introduction, qui regroupent les communications données lors de deux cycles de conférences.

On s'arrêtera plus longuement sur ces deux textes introductifs qui posent les fondations d'une réflexion très riche sur les usages éminemment conscients des citations empruntées à des auteurs anciens considérés comme des « autorités », réflexion qui souhaite se poursuivre au-delà de ce volume avec d'autres cycles de conférences, selon les auteurs.

Comme le souligne Maria Teresa Schettino dans son bref Avant-propos, un retour sur la notion d'auctoritas dans le domaine politique, avant d'aborder le domaine littéraire, s'avère nécessaire. En la définissant comme découlant « d'un processus d'auto-construction de sa propre personnalité politique autour de valeurs et de vertus partagées et reconnaissables par tous » (p. Io), l'auteur rappelle à quel point la légitimation du pouvoir, grâce à l'auctoritas, était une question centrale dans la réflexion politique antique. Toutefois, bien que la notion politique d'auctoritas ne puisse être dissociée de l'autorité littéraire, elle est moins présente dans les contributions réunies dans l'ouvrage, ainsi que le note l'auteur.

L'introduction complète et vient enrichir la réflexion liminaire sur la notion d'auctoritas. En esquissant un rappel historique de la mise en œuvre dans le temps de cette notion, Céline UrlacherBecht rappelle le rôle fondamental des Lumières et des écrits philosophiques dans la déconnexion que l'on peut observer entre l'autorité et le régime de la tradition. Elle enchaîne sur la crise profonde qui s'est fait jour dans notre rapport à la tradition au seuil de la modernité, crise qui se voit non seulement dans les remises en cause de l'autorité des Anciens, mais également dans le désamour actuel des formations qui transmettent les textes anciens. On ne peut que suivre l'auteur lorsqu'elle affirme avec conviction que les Anciens ont toujours beaucoup à nous apprendre.

Les contributions de la première partie, au nombre de quatre, portent sur l'Antiquité tardive et s'intéressent à la manière dont les Anciens font l'objet de citations dont les modifications de signification, voire les interpolations, méritent que l'on s'y arrête. Dans l'étude de Christophe Guignard, l'autorité dont jouissait Homère $\mathrm{au} \mathrm{III}{ }^{\mathrm{e}}$ siècle permet de montrer que le père des poètes était encore une référence pour les auteurs. Margarida Maria de Carvalho s'intéresse quant à elle à la construction du mythe attaché à Julien et à sa prétendue restauration païenne qui trouverait son fondement dans deux discours de Grégoire de Nazianze. Michel Cutino montre avec conviction qu'Ambroise cite Cicéron en détournant l'autorité des Anciens. La première partie s'achève sur la contribution de Giuseppe Zecchini qui s'attache à l'étude de toutes les citations présentes dans les Historiae adversus paganos d'Orose au $\mathrm{v}^{\mathrm{e}}$ siècle, montrant à son tour des exemples de détournement de l'autorité des Anciens.

La seconde partie regroupe cinq études portant sur les historiens antiques et l'autorité dont ils bénéficient à l'époque humaniste. Marie-Laure Freyburger analyse la manière dont Dion Cassius fut transmis par le courant humaniste; Céline Urlacher-Becht s'intéresse à une édition 
humaniste d'une compilation de TiteLive, ne retenant que les prodiges, établie par Julius Obsequens; Olivier Guerrier étudie l'utilisation de Plutarque comme " historien » dans l'humanisme français et Lucie Claire analyse la notion de prudentia à la Renaissance et la construction progressive de Tacite en "maître de la prudentia».

Ces quatre contributions suivent la première qu'il est nécessaire de traiter séparément, dans la mesure où Pascal Payen offre « une mise en perspective » en étudiant les historiens anciens comme autorités entre le $\mathrm{xv}^{\mathrm{e}}$ et $\mathrm{xIx}^{\mathrm{e}}$ siècles. Cette contribution, comme le précise l'auteur, synthétise plusieurs travaux déjà publiés et s'intéresse aux historiens anciens (historiens étant compris dans un sens très large) en tant que " détenteurs de vérité » qui ont été érigés, au fil des siècles, en figures d'autorité par des auteurs postérieurs. L'auteur souligne non seulement l'importance des traductions, qui permettent à la fois d'offrir les textes au public et de les transmettre, mais aussi les deux cas emblématiques que représentent Hérodote et Thucydide dans l'historiographie moderne.

Catherine PsiLakis

Université Lyon I catherine.psilakis@univ-lyonı.fr

Guy G. Stroumsa,

Religions d'Abraham : histoires croisées, Genève, Labor et Fides, 20I7, 346 p., 24 euros / ISBN 978-2-83og-I637-9.

Depuis plusieurs années, Guy G. Stroumsa [désormais S.] a contribué plus que quiconque à mettre en relation les chercheurs impliqués dans l'étude des cultures et des religions de l'espace méditerranéen dans l'Antiquité tardive, alors même que ses ouvrages eussent pu être classés dans des champs académiques différents ${ }^{8}$. Le présent livre Religions d'Abraham: histoires croisées constitue en quelque sorte le testament scientifique annoncé comme tel par l'auteur (p.9) et aborde les religions abrahamiques à travers la notion de connected stories afin de " décrire de façon succincte la dialectique complexe des échanges intellectuels, culturels, cultuels et sociaux entre les communautés des trois grandes religions monothéistes se prévalant de leur origine abrahamique » (p. 9).

Toutefois, le lecteur familier et attentif remarquera sinon l'identité, du moins la forte similitude entre les chapitres de l'ouvrage et la production antérieure de S., sans que cela ne vienne démentir l'intérêt et la qualité du livre qui ne constitue pas un simple résumé ou collage de travaux précédents, mais, pour reprendre le terme employé par S., une épitomé dont le caractère inédit repose sur la mise en relation des études entre elles ${ }^{9}$.

Dans l'introduction "Parcours d'un flâneur » (p. II-36), S. étudie les raisons qui l'ont poussé à devenir historien des

8 En témoignent Graham N. Stanton, Guy G. Stroumsa (éd.), Tolerance and Intolerance in Early Judaism and Christianity, Cambridge, 1998 ; David Shulman, Guy G. Stroumsa (éd.), Dream Cultures: Towards a Comparative History of Dreaming, Oxford, 1999 ; ou encore Margalit Finkelberg, Guy G. Stroumsa (éd.), Homer, the Bible, and Beyond. Literary and Religious Canons in the Ancient World, Leiden, 2003 ; Guy G. Stroumsa, La fin du sacrifice. Les mutations religieuses de l'Antiquité tardive, Paris, 2005.

9 Le lecteur trouvera dans la section “Sources » placée à la fin de l'ouvrage les références aux travaux originaux sur la base desquels Guy Stroumsa a écrit ce livre. 
religions et, à travers cet « itinéraire » (p. I2), cherche à mettre au jour la légitimité de cette discipline en se plaçant non pas sur le plan académique ou scientifique, mais sur celui de l'évènement ou plutôt des évènements qui ont façonné une recherche. Écrit peu après les attentats qui ont frappé la France en janvier 2015 et envoyé à quelques amis, ce texte se signale tant par la mise à nu d'un homme face à son lecteur que par la résonance du propos tenu : chaque homme est formé par " toute une série de brisures et de détours culturels, linguistiques et géographiques, à la fois par la philosophie et la religion » (p. I 7 ).

Évoquant la prégnance de la guerre dans sa vie, de son urgence et de son horreur, S. souligne aussi l'importance de sa formation à l'École Normale Israélite Orientale à Paris dans la prise de conscience de sa judéité, puis à l'Université de Jérusalem pour sa formation intellectuelle. Affirmant son intérêt pour l'histoire comparée des religions, il se heurte à la réaction amusée de ses amis qui y voient une pseudo-discipline tout juste bonne pour le dilettante. Il complète le tableau de sa formation en insistant sur son séjour à l'École Pratique des Hautes Études, où il étudie la première mythologie gnostique avec Michel Tardieu, et sa rencontre avec Peter Brown à Harvard.

Constatant les changements opérés dans l'université aujourd'hui, qui se rapproche dangereusement du statut d'entreprise “Soumises aux lois du marché »(p.28), S. décrit la période durant laquelle il enseigna comme un âge d'or dans lequel le c flâneur » peut étudier à l'envi les sujets qui l'intéressent: du manichéisme au christianisme de l'Antiquité tardive, des textes gnostiques coptes à l'orthodoxie chrétienne, S. considère chaque élément comme la partie du tout qu'il faut approcher comme tel. J'attire l'attention du lecteur sur deux points importants soulevés par S. : l'existence de groupes judéo-chrétiens après le Iv $^{\mathrm{e}}$ siècle et, surtout, les présupposés idéologiques qui dénaturent la recherche dans le champ de l'histoire des religions. Ayant déjà relevé ce problème à propos de Gershom Scholem (p. 2o), il explique que « rares sont ceux qui travaillent sur leur propre religion comme si c'était la religion des autres - et sur celles des autres comme si c'était la leur...» (p.3o), renouvelant la nécessité pour l'historien d'adopter l'approche sinon la plus objective, du moins la plus scientifique vis-à-vis de son objet.

Dans cette perspective, S. réitère les précautions que doit prendre l'historien : le refus de la recherche illusoire de la méthode, de la téléologie et la célébration de la force du hasard, faisant écho à la nature même de l'histoire des religions : un espace de réflexion où cohabitent plusieurs disciplines.

Dans le chapitre I « Religion d'Abraham et religions abrahamiques ", S., reprenant la conférence qu'il a prononcée lors de l'inauguration de la chaire d'études des "religions abrahamiques », cherche à “mettre en lumière les mécanismes par lesquels les religions naissent, évoluent, se développent, se transforment, impactent la société et, comme toutes les autres formes de réalités sociales, meurent» (p.39). Au cours de cet article liminaire, $\mathrm{S}$. se livre à une archéologie de l'histoire des religions, c'est-à-dire de l'étude des religions en tant que fait social, qu'il faut distinguer de la théologie qui se désintéresse « de la diversité des expériences humaines et de l'exercice de la pensée rationnelle » (p. 39). À cet égard, l'histoire des religions est avant tout comparatiste comme l'illustre le titre de l'ouvrage de $\mathrm{S}$. et la création de la chaire dédiée à Oxford.

S. revient dans le chapitre 2 “Trois anneaux ou trois imposteurs? Comparer le christianisme, le judaïsme et l'islam » sur l'histoire du comparatisme et de l'étude du “triangle théologique» constitué par le christianisme, le judaïsme et l'islam (p. 63-87). Centrée sur la figure d'Abraham (p. 42-46), la “ religion d'Abraham » est dès l'Antiquité conceptualisée comme étant la 
pratique de la religion pure que le judaïsme, le christianisme et l'islam revendiquent. En revanche, le concept de "religions abrahamiques ", né au $\mathrm{xx}^{\mathrm{e}}$ siècle, témoigne $\mathrm{du}$ changement de paradigme et de la volonté d'étendre les racines européennes afin de favoriser l'intégration culturelle des nouvelles populations musulmanes (p. 55). Dans une perspective plus large, le comparatisme doit amener à une meilleure compréhension des racines communes des religions (p.57-6r). À cet égard, le chapitre 9 " La religion impensable et la naissance de l'histoire des religions" prolonge la réflexion sur la naissance du concept moderne de "religion» à partir de la tentative de définition religieuse des populations d'Amérique du Sud après les découvertes de Christophe Colomb (p. 22I-239).

Dans le chapitre 3, S. étudie les mythes des origines en mettant en exergue comment les auteurs chrétiens participent de la transformation radicale des perceptions anthropologiques à partir d'une réflexion sur les textes bibliques et, notamment, sur l'expulsion d'Adam hors de l'Éden. Constatant l'absence de texte unique d'un mythe “ officiel » des origines, les auteurs chrétiens conçoivent l'origine à partir du récit adamique. Dans la perspective "pessimiste» des premiers temps de l'humanité, seule la venue d'un nouvel Adam peut sauver l'humanité : l'expulsion n'est que temporaire, et non intrinsèque à la nature humaine. De plus, là où le début de l'humanité n'est qu'un prélude à la naissance d'Israël et au développement d'une histoire sainte au cours de laquelle l'accent est mis sur les racines historiques et l'identité du peuple juif “ élu », les auteurs chrétiens perçoivent le christianisme comme le uerus Israel et Adam n'est qu'une préfiguration du Christ. Cette dichotomie se retrouve dans l'union des deux Testaments autour du problème de la figure et de la réalisation.

Les chapitres 5 «Écriture et autorité : la transformation chrétienne de la culture antique» et 6 “Juifs et chrétiens dans l'Antiquité tardive» sont consacrés aux transformations religieuses dans l'Antiquité tardive $^{10}$. L'une des caractéristiques principales est la création d'une nouvelle paideia, différente de l'ancienne fondée sur les auteurs classiques et les artes liberales, par l'utilisation des textes sacrés dans la formation et l'éducation chrétienne. S. revient aussi sur les deux attitudes différentes des chrétiens à l'égard de la paideia traditionnelle, entre position "fidéiste " rejetant totalement les œuvres païennes et théorie de la double helix défendue par saint Augustin dans le De doctrina christiana ${ }^{11}$.

L'autre grande transformation s'effectue dans le domaine théologico-politique. En effet, les juifs et les chrétiens suivent une trajectoire inverse: de communauté “protégée »-même s'il faut nuancer cette idée - les juifs deviennent de plus en plus isolés au sein de l'empire gréco-romain et sont placés dans la même catégorie que les sectes hérétiques (en témoigne le livre XVI du Code Théodosien); au contraire,

10 À ce propos, voir Guy G. Stroumsa, "The End of Sacrifice: Religious Mutations of Late Antiquity ", dans Johann P. Arnason, Kurt A. Raaflaub (éd.), The Roman Empire in Context: Historical and Comparative Perspectives, London, p. 134-147.

11 Voir Guy G. Stroumsa, « Scriptures and paideia in Late Antiquity ", dans M. R. Niehoff (éd.), Homer and the Bible in the Eyes of Ancient Interpreters, Leiden, 2012, p. 29-41; Id., "Écritures et paideia dans l'Antiquité tardive », dans M. Tardieu, M. Gorea (éd.), Autorité des auteurs antiques: entre anonymat, masque et authenticité, Turnhout, 2014, p. 189-202; Id., " The New Self and Reading Practices in Late Antique Christianity ", Church History and Religious Culture 95, 2015, p. 1-18. 
les chrétiens changent de statut, passant de celui de secte persécutée à religion officielle. Alors que l'isolement progressif de la communauté juive permet au mouvement rabbinique d'imposer progressivement son autorité sur les croyants ${ }^{12}$, l'avènement du christianisme en tant que religion dominante et officielle implique une modification de la pratique religieuse qui ne relève plus de la sphère civique (comme les Grandes Panathénées), mais de la sphère communautaire.

Le chapitre 4 “Intolérance religieuse et christianisme : remarques sur un paradoxe » est consacré à l'intolérance religieuse qui, comme le souligne S., ne manque pas de faire écho à notre actualité et de façonner notre approche et notre compréhension des temps anciens (p. II3). S. propose un résumé des travaux sur le sujet en mettant en exergue le passage de l'exclusivisme juif à l'intolérance chrétienne (p. II9-I29), notamment à partir du $\mathrm{IV}^{\mathrm{e}}$ siècle où la progression du christianisme se traduit par une violence antijuive accrue dans le discours chrétien - en témoignent les homélies aduersus Iudaeos de Jean Chrysostome et l'œuvre de Cyrille d'Alexandrie. S. revient aussi sur l'image d'Épinal d'un paganisme tolérant érigé comme barrière contre l'intolérance chrétienne, image qui repose sur une mécompréhension de la religio grécoromaine, c'est-à-dire une religion civile

12 Voir par exemple Simon Mimouni, "Le judaïsme 'sacerdotal et synagogal' dans le Code Théodosien », dans C. Bonnet, F. Briquel-Chatonnet (éds.), Ekklèsia. Approches croisées d'histoire politique et religieuse. Mélanges offerts à Marie-Françoise Baslez, Toulouse, 2017, 155-167 ; Id., L. Painchaud (éds.), La sacerdotalisation dans le judaïme synagogal, dans le christianisme et dans le rabbinisme. Actes du colloque de Québec, 18-20 septembre 2014, Turnhout, 2018. reposant sur des valeurs et des traditions communes, qui se distingue de la religio chrétienne, dynamique, dont le cœur est la vérité révélée.

Le chapitre 7 «Barbares ou hérétiques? Juifs et arabes dans la conscience byzantine» porte plus spécifiquement sur l'évolution du terme barbaros à la lumière de la comparaison entre la perception des juifs et des Arabes dans l'empire romain christianisé. Pourtant, les chrétiens, en tant que descendants des juifs, sont euxmêmes barbaroi! S. insiste sur le fait que “ chrétien » devient une nouvelle catégorie dont dépend une nouvelle "sagesse barbare» (p. I72) et dont les membres ne reconnaissent pas les catégories traditionnelles, mais mettent en avant l'opposition entre " chrétien » (croyant) et apistoi : d'une part, entre "chrétien " et hellênes, d'autre part entre "chrétien " et mauvais croyant (les juifs par exemple). De cette hérésiologie découle la caractérisation de l'islam à partir de Jean Damascène.

Dans le chapitre 8 “ Judéo-christianisme et origines de l'islam », S. revient sur le problème posé par le concept de “judéochristianisme» et s'oppose à Daniel Boyarin partisan de son abandon ${ }^{13}$ (p. IgoI94), puis discute l'élargissement du concept d' “Antiquité tardive » (p. 195-ıg8), notamment par l'importance accordée à l'étude de la péninsule arabique ${ }^{14}$ (p. 199206). À ce propos, S. s'intéresse surtout à l'existence d'un courant abrahamique

13 Daniel Boyarin, “Rethinking JewishChristianity: An Argument for Dismantling a Dubious Category (to which is Appended a Correction of my Border Lines), Jewish Quarterly Review 99, 2009, p. 7-36.

14 Voir par exemple Glen Bowersock, The Throne of Adulis: Red Sea Wars on the Eve of Islam, Oxford, 2013; Id., The Crucible of Islam, Cambridge (Mass.), 2017. 
pré-islamique qui s'inscrit dans la pensée que l'islam, à l'origine, constitue une communauté regroupant l'ensemble des monothéistes ${ }^{15}$ rassemblés autour de la figure d'Abraham, sorte de héros culturel dépassant les limites de la communauté juive, et revendiquant la pratique pure de la religion. Cette hypothèse repose certes sur de nombreux témoignages anciens, mais S. note qu'elle « ne peut être démontrée en l'état actuel de nos connaissances. Un tel mouvement se serait situé aux marges à la fois du judaïsme et du christianisme, de façon similaire aux judéo-chrétiens » (p. 2I7). S. préfère mettre en avant l'idée d'une praeparatio coranica supportée par les judéo-chrétiens : “ la filière judéochrétienne s'impose comme ayant offert un levain exceptionnel, permettant ainsi au message de Mohammed de cristalliser, dans le riche terroir des traditions et attitudes religieuses de l'Antiquité tardive » (p. 2I9).

Les chapitres Io, II et I2 sont des uaria qui s'insèrent peu ou prou dans l'ensemble de l'ouvrage. Au chapitre io “John Selden et les origines de l'orientalisme », S. revient sur la formation de John Selden, juriste anglais né en i585, ainsi que sur le contexte culturel et intellectuel dans lequel s'inscrit sa pensée d' " antiquaire », c'est-à-dire ceux dont l'intérêt pour les langues, les religions et les cultures du Proche-Orient ne relève pas de la coquetterie, mais de l'amour de la culture, et qui constituent un groupe de précurseurs de l'histoire des religions. Après être revenu au chapitre II sur la diffusion du mythe du Déluge aux xvir ${ }^{\mathrm{e}}$ $\mathrm{XVIII}^{\mathrm{e}}$ siècles comme indice de l'importance croissante de l'orientalisme, S. revient au chapitre I2, intitulé " Homère et la Bible », sur les liens entre le poète grec épique et les Écritures.

15 Fred Donner, Muhammad and the Believers: at the Origins of Islam, Cambridge (Mass.), 2010.
Le dernier chapitre “Tentations du christianisme: Henri Bergson, Simone Weil, Emmanuel Levinas » propose trois exemples d'intellectuels juifs pris par trois tentations différentes pour le christianisme. S. rappelle que Simone Weil connait bien l'œuvre d'Henri Bergson ${ }^{16}$ et qu'Emmanuel Levinas s'oppose à l'attitude "gnostique " de la philosophie vis-à-vis de la Bible hébraïque ${ }^{17}$. Dans le cas des deux premiers cités, la tentation s'inscrit dans leur intérêt profond pour l'histoire des religions (p. 299) perçue comme le dévoilement de la vérité. Contrairement à Simone Weil, engagée politiquement pour la cause ouvrière et dans la guerre civile espagnole, Henri Bergson se tient à l'écart de toute forme de révolution... sauf dans le cas de la religion: la seule forme de religion qui l'attire est celle qui transforme l'humanité, la religion révolutionnaire. Contrairement à la conception bergsonienne de l'évolution historique des religions jusqu'au dynamisme de la religion ouverte telle qu'elle s'épanouit dans le christianisme, Simone Weil soutient l'idée d'une philosophia perennis, éternelle et statique (p.3og-3i6). De côté, un temps tenté par le christianisme, en tant qu'il est considéré comme vecteur de valeurs universelles, Emmanuel Levinas s'en détourne après la Shoah. Revenant sur l'émergence du christianisme, il souligne que les grandes idées chrétiennes sont déjà présentes dans le judaïsme; en cela, «il comprend l'élection d'Israël selon l'ordre de l'éthique, plutôt que du théologique» (p. 325).

Le lecteur, qui garde en mémoire la tentation du christianisme de trois

16 Voir par exemple Martin Buber, “ The Silent Question », dans M. Buber, On Judaism, New York [Schocken], 1995 [1967], pp. 202-213.

17 Emmanuel Levinas, "Simone Weil contre la Bible ", dans Difficile liberté, Paris, 1976 [1967], pp. 205-217. 
intellectuels juifs, influencée par le sentiment de l'horreur de la guerre, ainsi que les références aux différentes formes d'intolérance et de violence religieuses, se voit rappeler dans l' “ Envoi » la nécessaire subversion de l'histoire des religions: "le courage civique devient un devoir intellectuel et le courage intellectuel un devoir civique » pour l'humanité (p. 338).

Pour finir, je signalerai deux errata dans la version finale: “ comme l'étude religions abrahamiques » (p. 38), “les fils de noé » (p. 346).

David LORIN EPHE- PSL University Research / La Sapienza - Università davidlorinz@gmail.com

Jean Yvonneau (éd.), La Muse au long couteau. Critias, de la création littéraire au terrorisme d'État, Bordeaux, Ausonius Éditions, Scripta Antiqua I07, 2018, 2I6 p., 25 euros/ IsSN I298-I99o.

Le recueil dont Jean Yvonneau a assuré l'édition réunit la majorité des communications du colloque international et interdisciplinaire qui s'est tenu à Bordeaux, les 23 et 24 octobre 2009, autour de Critias, un des Trente Tyrans qui ont sévi à Athènes au lendemain de la guerre du Péloponnèse et dont Xénophon a retracé les exactions dans les Helléniques. Sous ce titre évocateur La Muse au long couteau, il a pour finalité d'explorer la personnalité de ce personnage, oncle de Platon, familier de Socrate et d'Alcibiade, et de comprendre la pensée politique d'un des plus farouches ennemis de la démocratie. Critias n'a pas seulement intéressé les historiens. Poète et philosophe, il a retenu l'attention, au xix ${ }^{\mathrm{e}}$ siècle, des philologues qui ont reconnu sa valeur littéraire. Les huit contributions sont regroupées autour de cinq thématiques : la redécouverte, le poète, la pensée, l'homme politique et la postérité. Chacune d'elles est suivie d'une bibliographie et est résumée, en français et en anglais, en fin d'ouvrage après l'index des sources.

Dans son étude intitulée "Critias : l'invention et l'inventaire»(p. I3-32), Jean Yvonneau présente la production littéraire de Critias, qui aurait composé entre io et 2I ouvrages. En dépit du renouveau de la philologie au $\mathrm{xIx}^{\mathrm{e}}$ siècle, l'étude de ses œuvres reste difficile en raison de l'état fragmentaire des sources et de la passion qu'a suscitée le personnage auquel on accorde trop ou trop peu: "Critias aimante mais n'offre point de boussole» (p. 27). Critias fut un auteur reconnu et les fragments qui lui sont attribués, en poésie et en prose, prouvent la richesse de son œuvre, de l'élégie aux constitutions. Qualifié de polygraphe, il serait en effet le premier auteur des Politeiai. Si une Constitution des Lacédémoniens lui est attribuée, la question reste toujours posée de savoir s'il est bien le "Vieil Oligarque », auteur de la Constitution des Athéniens. Gabriele Burzacchini propose une étude philologique stimulante de sa poésie élégiaque. Après l'établissement de chacun des fragments, il donne une traduction et un commentaire enrichi des différentes hypothèses interprétatives (p. 35-59). Il souligne les traits novateurs, voire modernes, de la poésie de Critias. Il relève son ironie, en particulier à propos d'Alcibiade, de 416 à 406 , date de son exil en Thessalie. Les fragments les plus longs, connus grâce au Banquet des Sophistes d'Athénée, font l'éloge de la sobriété des Spartiates opposée aux banquets des Athéniens et donnent le catalogue des inventions et spécialités des différentes régions de la Grèce, du jeu du cottabe imputé aux Siciliens à l'invention de l'écriture par les Phéniciens. Critias fut aussi un auteur de théâtre talentueux, puisqu'il fut assimilé à Euripide. Alessandro Boschi (p. 6I-79) s'intéresse à deux fragments de la parodos de la tragédie Pirithoos qu'Athénée 
de Naucratis attribua à Critias. Dans le premier, il suggère une correction qui substitue aux constellations des Ourses celle des Gémeaux et il voit le Soleil en l'être " engendré par lui-même », que " la foule innombrable d'étoiles environne sans cesse de danses ", image illustrée par le lécythe attique du Peintre de Sappho (p. 75). Comme la tragédie se situait dans le monde infernal, la parodos représentait “une trouée céleste et astrale ». Pour l'étude de la pensée de Critias, Fritz-Gregor Hermann (p. 83-in5) ouvre le dossier de l'influence qu'il a exercée sur son neveu Platon, tant pour les concepts, la terminologie que pour les idées. Platon fait en effet intervenir Critias dès ses premiers dialogues socratiques jusqu'aux Lois. Sa place dans le Charmide et son portrait concordent avec ce que nous apprennent les fragments sur ses valeurs et son intérêt pour Sparte. Dans la République, Platon dénonce en son oncle non seulement une sorte d'antiSocrate mais aussi un sycophante. En traquant les superlatifs et les comparatifs dans l'œuvre de Critias, Emmanuèle Caire éclaire « l'archéologie de sa conception de l'excellence » (p. II7) qui aboutit à la tyrannie qu'il institua en 404-403 et dont témoigne le conflit qui l'opposa à Théramène (p. II7-I36). Que ce soit en Thessalie ou à Lacédémone, Critias met toujours l'accent sur les inventions qui valorisent le génie des inventeurs et les qualités de leur cité. Autre point de sa pensée : l'entraînement est primordial, car il est la condition d'une hygiène $\mathrm{du}$ comportement qui forme “ des athlètes du corps, des athlètes de la technique et des athlètes de l'esprit »(p. i25). De plus, en analysant l'extrait de Sisyphe, considéré comme le premier manifeste de l'athéisme, Emmanuèle Caire souligne combien la religion est pour Critias à mettre au crédit d'un inventeur “à l'intelligence ferme et subtile », puisqu'il a donné aux régimes autoritaires le moyen de contrôler les esprits. Pour le tyran, la compétition (agôn) est au cœur du jeu politique d'où son opposition à Théramène. Les beltistoi dont il fait partie doivent non seulement combattre en permanence les ennemis de la patrie mais aussi leurs rivaux dans la cité. Critias et son entourage méritent toute l'attention de Pierre Brulé et Jérôme Wilgaux. Le premier étudie le laconisme; le second les réseaux de solidarités dans l'Athènes de la fin du v viècle (p. I39-i58). Les Laconisants se distinguaient par leur vêtement, leur apparence physique et leur admiration pour Sparte, mais cette posture sociale n'était pas l'apanage des aristocrates dans l'Athènes démocratique. Pierre Brulé souligne par ailleurs combien la tyrannie de Critias, loin de représenter le triomphe de la laconomanie en 404-403, en sonna le glas sur le plan politique (p. I46), tandis que naissait une réflexion philosophique qui a fait de Sparte un des modèles possibles pour penser la cité idéale. Jérôme Wilgaux conteste que les Trente aient été l'expression d'une élite homogène par sa fortune et son positionnement politique. Malgré la rareté des sources, les Tyrans identifiables ont pour traits communs d'avoir exercé des responsabilités dans les institutions démocratiques, même quand ils n'appartenaient pas à la même génération. Plus que les alliances familiales et les réseaux de solidarité qui se faisaient et se défaisaient en fonction des circonstances, comptaient les choix individuels. Il n'y a ni logique de “classe », ni logique familiale. Théramène et Critias étaient proches sociologiquement par la fortune, la naissance et l'éducation, mais ils se sont opposés. Quant à Platon, neveu de Critias, il fut si indigné par les exactions des Trente qu'il préféra se dissocier des crimes qu'ils avaient commis. En préalable de sa communication (p. 159 -I75), Anton Powell cherche à se dédouaner de tout penchant pour Critias et sa politique. Il va jusqu'à se demander s'il lui est possible de le comprendre en raison du "dégoût » qu'il lui inspire. Il voit une analogie entre le laconisme de Critias et l'admiration aveugle 
de certains intellectuels de Cambridge (et d'ailleurs) pour le modèle soviétique dans les années 30 ou, plus tard, pour la Chine de Mao. À chaque époque son “mirage». Pour lui, l'admiration de Critias pour Sparte s'explique par le rôle qu'elle a joué dans les guerres médiques et par la victoire qu'elle a remportée en 404. En confrontant le récit de Xénophon dans les Helléniques et le Contre Ératosthène de Lysias, il met en lumière les responsabilités partagées des Trente et des Spartiates dans l'installation et l'exercice de la tyrannie. Les institutions, et particulièrement l'éphorat, n'ont pu être mises en place dans l'Athènes occupée qu'avec l'accord de Lysandre, mais il ne s'agissait que d'un leurre dont Critias fut en fait la dupe. Dans la dernière partie intitulée “ la postérité », Sophie Gotteland étudie l'image de Critias dans la Seconde Sophistique et les traités des rhéteurs d'époque impériale (p. I79-ı96). En raison d'une damnatio memoriae, Critias n'est pas mentionné dans la Constitution des Athéniens. Pourtant il mérite une narration, “car peu nombreux sont ceux qui savent ce qu'il a fait », reconnaît Aristote dans la Rhétorique. Quelques siècles plus tard, au temps d'Hérode Atticus, Critias suscite de nouveau l'intérêt en raison de ses qualités stylistiques (Philostrate) et de sa relation avec Socrate. Si Critias, dans les déclamations (Hermogène, Aelius Aristide, Libanios), incarne un régime honni, dépourvu de toute morale, il est aussi présenté comme le disciple dissident de Socrate, celui dont la nature ruine toute pédagogie (Plutarque). Parler de Critias donne l'occasion aux auteurs de développer un contre-modèle qui valorise les valeurs de l'hellénisme.

La Muse au long couteau a plus d'une facette. Grâce à cet ouvrage, d'une facture élégante et d'une lecture agréable, le personnage de Critias gagne en profondeur et en cohérence. L'originalité et la richesse de sa pensée sont soulignées par la présentation des fragments de son œuvre, établis, traduits et commentés sous différents angles, philologiques, philosophiques et historiques. Au fil des contributions s'esquisse l'archéologie d'une idéologie dont le contexte est bien connu, mais dont on perçoit mieux la logique. Il est évident que Critias ne doit pas être réduit à l'image du tyran sanguinaire. Son itinéraire ne peut être correctement analysé que si l'on prend la mesure de l'influence qu'il a exercée sur ses contemporains. Le poète et philosophe qu'il fut sont inséparables de l'homme politique qu'il devint. Avant d'être un des Trente et le bras armé des Spartiates, Critias représenta un courant de pensée qui fit la richesse de la vie intellectuelle et politique athénienne dans la seconde moitié du v $\mathrm{v}^{\mathrm{e}}$ siècle. Ce recueil a le mérite de le rappeler.

Geneviève Hoffmann Université de Picardie Jules Verne genevieve.hoffmann@wanadoo.fr 